\title{
The Araucaria project. Bright variable stars in NGC 6822 from a wide-field imaging survey ${ }^{\star}, \star \star$
}

\author{
R. E. Mennickent ${ }^{1}$, W. Gieren ${ }^{1}$, I. Soszyński ${ }^{1,2}$, and G. Pietrzyński ${ }^{1,2}$ \\ 1 Universidad de Concepción, Departamento de Física, Casilla 160-C, Concepción, Chile \\ e-mail: [rmennick; soszynsk;wgieren]@astro-udec.cl; pietrzyn@hubble.cfm.udec.cl \\ 2 Warsaw University Observatory, Al. Ujazdowskie 4, 00-478 Warsaw, Poland
}

Received 14 October 2005 / Accepted 5 January 2006

\begin{abstract}
We have performed a search for variable stars in the dwarf irregular galaxy NGC 6822 using wide-field multi-epoch VI photometry down to a limiting magnitude $V \sim 22$. Apart from the Cepheid variables in this galaxy already reported in an earlier paper by Pietrzynski et al. (2004), we have found 1019 "non-periodic" variable stars, 50 periodically variable stars with periods ranging from 0.12 to 66 days and 146 probably periodic variables. Twelve of these stars are eclipsing binaries and fifteen are likely new, low-amplitude Cepheids. Interestingly, seven of these Cepheid candidates have periods longer than 100 days, have very low amplitudes (less than 0.2 mag in I), and are very red. They could be young, massive Cepheids still embedded in dusty envelopes. The other objects span a huge range in colours and represent a mixture of different types of luminous variables. Many of the variables classified as non-periodic in the present study may turn out to be periodic variables once a much longer time baseline will be available to study them.
\end{abstract}

We provide the catalogue of photometric parameters and show the atlas of light curves for the new variable stars. Our present catalogue is complementary to the one of Baldacci et al. (2005) which has focussed on very short-period and fainter variables in a subfield in NGC 6822.

Key words. galaxies: stellar content - binaries: eclipsing - stars: variables: general - Cepheids - Local Group - binaries: general

\section{Introduction}

NGC 6822 is a relatively well studied dwarf irregular galaxy of the Local Group. In recent years its content of variable stars has been investigated by several authors, mainly with emphasis on distance indicators, like Cepheids and RR Lyrae stars, and short period variables. Antonello et al. (2002a,b) discovered 130 variable stars in NGC 6822, 21 of them Cepheids, 18 other periodic variables and 91 irregular or semiregular variables. Their study was based on a 3-year monitoring of a small $(3.8 \times 3.8$ arcmin) field of NGC 6822 . In a series of papers, Clementini et al. (2003), Baldacci et al. (2004a,b), and Baldacci et al. (2005), using deep VLT images of a field of $6.8 \times 6.8$ arcmin, reported 390 candidate variables. They used data taken on 3 half-nights distributed over 5 days in August 2001. They classified the variables in Main Sequence Variables (MSV, 36 stars), Classical Instability Strip Variables (CISV, 160 stars) and Red Giant Branch Variables (RGBV, 66 stars). They also found 6 eclipsing binaries. As they noted, their data

* Complete Tables 2 and 3 are only available in electronic form at the CDS via anonymous ftp to cdsarc.u-strasbg.fr (130.79.128.5) or via

http://cdsweb.u-strasbg.fr/cgi-bin/qcat? J/A+A/450/873

$\star \star$ Complete Figs. 1 and 2 are only available in electronic form at http://www. edpsciences.org distribution was optimized for searching periods in the range of 0.2-0.7 days. On the other hand, Pietrzyński et al. (2004, hereafter P04) monitored the whole galaxy in the $V$ and $I$ bands during 77 nights, spread over nearly one year, with the aid of mosaic images $(35 \times 35$ squared arcmin $)$ taken with the Warsaw $1.3 \mathrm{~m}$ telescope at Las Campanas Observatory, discovering 116 Cepheids with periods ranging from 1.7 to 124 days. These authors determined, using the reddening-free Wesenheit index, a distance modulus for NGC 6822 of 23.34 ( \pm 0.04 statistical and \pm 0.05 systematic) mag. In the present paper, we use the photometric database provided by $\mathrm{P} 04$ to search for variable stars in NGC 6822. The investigation presented in this paper complements the previous ones since it is the first simultaneous monitoring of the whole galaxy during a time interval of almost one year, allowing us to detect and characterize the population of relatively long-period, bright variables from these images.

In Sect. 2, we give a review of the observations and explain our searching tools and selection methods. In Sect. 3, we show the results of our investigation, and discuss them in Sect. 4. Conclusions and future prospects are outlined in Sect. 5.

\section{Observations and selection of variable stars}

The CCD VI images presented in this paper were obtained with the Warsaw $1.3 \mathrm{~m}$ telescope at Las Campanas Observatory. 
Most of the observations were obtained in 2002 between July 3 and November 21. Additional observations were made during three photometric nights in 2003 May in order to accurately calibrate the data and improve the periods of the detected variable stars. The time baseline is about 330 days, total observing nights 77 and the typical separation between consecutive images is 1 or 2 days. Details on the observations, and on the adopted observing procedures can be found in P04. Our present search for variable stars was carried out on the $I$ filter dataset. All our magnitudes were calibrated and corrected for the differential photometric zero point across the entire field of view (P04). Color corrections were applied using the sum of foreground and intrinsic reddening for NGC 300, $E(B-V)=0.36$, derived by McGonegal et al. (1983) and the reddening law of Schlegel et al. (1988), viz. $A_{V}=3.24(B-V)$ and $A_{I}=1.96 E(B-V)$. The absolute magnitude $M_{I}$ was calculated using the distance modulus for NGC 6822 derived by P04, viz. $(m-M)_{0}=23.34 \mathrm{mag}$.

The I-band light curves of the more than one hundred thousand (107389) photometrically identified stars in NGC 6822 were extracted from our database, yielding robust estimates for the mean magnitude $\langle I\rangle$ and standard deviation $\sigma$. In order to achieve this step, a special algorithm was used which is less sensitive to outliers than the usual ones. The c-code is freely available at http://www.astro.lu.se/ stefans/aprog.html. We adopted the following procedure independently for each of the 8 chips of the $8 \mathrm{k} \times 8 \mathrm{k}$ detector (see details of the instrumentation in http://ogle.astrouw.edu.pl). The cloud of pairs $\sigma-\langle I\rangle$ were passed by a smoothing function $f(I)$ representing the standard deviation as a function of magnitude. The $f$ function turned to be approximately $0.007,0.008,0.018,0.05$, $0.10,0.22$ and $0.47 \mathrm{mag}$ for $I=16,17,18,19,20,21$ and $22 \mathrm{mag}$, respectively, almost independent of the chip number. Stars with $\sigma$ values larger than $1.1 f$ were picked out as variable star candidates. This procedure left us with 22631 stars, whose light curves were visually inspected in order to reject false detections due to artifacts of the analysys method. These artifact might depend on a number of effects, ranging from hot pixels to cosmic rays or blending of images (coupled with variable seeing). The light curves were also inspected for periodic variability using fast and reliable period-searching algorithms based on the algorithm of analysis of variance (SchwarzenbergCzerny 1989) and Discrete Fourier Transform. We calculated the parameter $S / N$, defined as the ratio between the amplitude of the highest peak in the periodogram and the noise amplitude, the latter one was calculated as the average of the amplitudes of all peaks in the periodogram. A safe criterion to establish a significant frequency seems to be $S / N>4$ (Breger et al. 1993; Aerts \& Kolenberg 2005). The figure is also an useful reference level to evaluate the level of variability detected in the stars, especially those showing "non-periodic" variability (see next section).

\section{Results}

In addition to the Cepheids already reported by P04, we found a total of 1019 "non-periodic" variable stars, 146 probably periodic variables and 50 periodically variable stars. Twelve of them are eclipsing binaries, with periods between 0.12 and 66 days. "Non-periodic" variables are those without a clear peak in the periodogram, but with a periodogram clearly not compatible with noise (i.e. with $S / N \gtrsim 4$ ). The stars classified as "probably periodic" presented a peak in the periodogram, but it is not well defined; for these stars, we were able to determine a time scale of variability, rather than a true periodicity. Many of these objects could be periodic, but the time baseline spanned by the present data is not long enough to decide this question unambiguously. On the other hand, periodic stars showed a clear peak in the periodogram and their periodicity is well established. Cross-correlating our sample stars with previous surveys in NGC 6822 we found three stars in common with Antonello et al. (2002a), viz. their stars V 0130 (1876-2), V 2881 (765-3) and V 1578 (5874-6), and two other stars in common with Baldacci et al. (2005); their stars V 112 (710-3) and V 215 (1363-3). For the periodic stars, we determined the ephemeris for the time of maxima or the main minimum in the case of eclipsing binaries. The catalogue of variable stars, containing their mean photometric properties and periods, is given in Tables 1-3. The phased light curves for the periodic variables are presented in Fig. 1. The light curves for the probably periodic variables are displayed in Fig. 2.

\section{Discussion}

\subsection{The periodic and probably-periodic variable stars}

Looking at the colour-magnitude diagram of the periodic and probably periodic variables (Fig. 3), we observe that they span a huge range of colours, from $V-I=-0.4$ to 2.7 . They are also quite luminous, with absolute visual magnitudes between -8.6 (the case of 32-5, the brightest star in our sample) and -2.6 (the star 5574-1). The stars seem to be placed in well-defined regions of the CM diagram. For instance, we distinguish five "blue" variable stars, which are inside the upper left rectangle in Fig. 3. These stars have relatively blue $V-I$ colours, 3 of them are placed close to the region of the O-type main sequence stars. In addition, 8 stars are placed above the yellow supergiant track, and 76 stars are red variables located in the asymptotic giant branch region indicated by the rectangle at the right side of the Fig. 3. Most of these later stars are very slow variables, with time scales of variability between 71 and 188 days, although the upper limit is severely biased by our time baseline. In addition, we have found a large percentage of our variables mostly occupying the upper part of the classical Cepheid instability strip, and a small fraction of stars near the late-K supergiant track. It is interesting to note that only about $1 / 3$ of the stars located in the classical Cepheid instability strip were found to be Cepheids, as indicated by their typical light curve shapes and colours. Contamination of the sample by foreground stars is not large enough to account for this effect (see Sect. 5), so probably there are other kinds of variable stars apart from Cepheids inside their pulsation instability zone (see also Fig. 7, and corresponding discussion in Sect. 4.2, for additional low amplitude variables in this region). 
Table 1. Periodically variable stars towards NGC 6822. We give the amplitude in the $I$-band $(\Delta I)$, period and absolute magnitude, dereddened colours, and $I$ and $V$ magnitudes. The error in the last digit of the period is given in parenthesis. An " $e$ " accompanying the period means the stars is an eclipsing binary. Times for maxima (or main minima, in the case of eclipsing binaries) are also given. $e$ (HJD) means error in HJD and $M A$ means "multiple aliases".

\begin{tabular}{|c|c|c|c|c|c|c|c|c|c|c|}
\hline ID & alpha(2000) & $\operatorname{Dec}(2000)$ & $\Delta I$ (mag) & $P$ (days) & $M_{I}$ & $(V-I)_{0}$ & $I$ & $V$ & HJD & $e(\mathrm{HJD})$ \\
\hline $291-6$ & $19: 43: 55.53$ & $-14: 43: 28.9$ & 0.70 & $0.29006(9) \mathrm{e}$ & -5.87 & 0.80 & 18.14 & 19.40 & 2459.0477 & 0.0014 \\
\hline $32-5$ & $19: 43: 58.13$ & $-14: 33: 11.8$ & 0.05 & $43(5)$ & -9.32 & 0.71 & 14.69 & 15.86 & 2474.4549 & 1.7200 \\
\hline $234-5$ & $19: 43: 58.92$ & $-14: 36: 38.1$ & 0.17 & $5.12(6)$ or $2.48(3)$ & -6.10 & 0.84 & 17.90 & 19.20 & 2459.1901 & 0.1024 \\
\hline $241-5$ & 19:44:00.77 & $-14: 34: 33.0$ & 0.69 & $0.2546(2) \mathrm{e}$ & -6.31 & 0.76 & 17.69 & 18.91 & 2459.3815 & 0.0013 \\
\hline $684-7$ & $19: 44: 02.19$ & $-14: 49: 56.4$ & 0.36 & $0.11935(10) \mathrm{MA}$ & -5.12 & 0.65 & 18.88 & 19.99 & 2458.9319 & 0.0036 \\
\hline $382-7$ & $19: 44: 02.92$ & $-14: 52: 00.6$ & 0.20 & $0.2760(5) \mathrm{MA}$ & -6.03 & 0.60 & 17.97 & 19.03 & 2459.0798 & 0.0072 \\
\hline $58-5$ & 19:44:07.15 & $-14: 31: 57.4$ & 0.10 & $2.76(3)$ & -8.94 & 0.57 & 15.07 & 16.10 & 2459.5427 & 0.1380 \\
\hline $112-7$ & $19: 44: 11.63$ & $-14: 55: 21.9$ & 0.09 & $4.51(3)$ & -7.78 & 1.19 & 16.22 & 17.87 & 2462.9592 & 0.1353 \\
\hline $377-6$ & 19:44:11.76 & $-14: 44: 12.5$ & 0.31 & $0.17858(8)$ & -6.19 & 0.08 & 17.82 & 18.36 & 2458.9957 & 0.0018 \\
\hline $1433-6$ & $19: 44: 12.78$ & $-14: 41: 08.3$ & 1.03 & $5.73(6)$ & -4.11 & 0.48 & 17.77 & 19.15 & 2460.6911 & 0.0860 \\
\hline $390-6$ & $19: 44: 13.72$ & $-14: 39: 47.1$ & 0.13 & $50(-3,+10)$ or $1.017(5)$ & -6.23 & 0.92 & 17.83 & 19.34 & 2489.9253 & 0.7500 \\
\hline $6220-5$ & $19: 44: 20.84$ & $-14: 31: 45.4$ & 0.14 & $0.11985(5) \mathrm{MA}$ & -6.18 & 1.05 & 16.21 & 17.42 & 2458.9402 & 0.0048 \\
\hline $6140-5$ & $19: 44: 26.22$ & $-14: 36: 24.4$ & 0.60 & $0.34216(5) \mathrm{e}$ & -7.80 & 0.75 & 18.52 & 20.08 & 2458.8904 & 0.0021 \\
\hline $4983-6$ & $19: 44: 27.80$ & $-14: 43: 00.0$ & 0.31 & $0.1470(1)$ or $0.0735(1)$ & -5.49 & 1.10 & 17.61 & 18.78 & 2458.9420 & 0.0029 \\
\hline $4786-6$ & $19: 44: 30.88$ & $-14: 40: 00.1$ & 0.11 & $3.745(15) \mathrm{MA}$ & -6.40 & 0.71 & 15.99 & 16.80 & 2461.7250 & 0.1498 \\
\hline $6066-5$ & $19: 44: 30.88$ & $-14: 38: 27.4$ & 0.31 & $8.34(15)$ & -8.02 & 0.35 & 18.37 & 18.50 & 2462.9778 & 0.1668 \\
\hline $4794-6$ & $19: 44: 32.83$ & $-14: 46: 01.1$ & 0.72 & $0.4972(6) \mathrm{e}$ & -6.57 & 0.40 & 16.71 & 18.59 & 2459.1496 & 0.0050 \\
\hline $4907-6$ & $19: 44: 34.24$ & $-14: 42: 20.5$ & 0.49 & $2.67(3)$ & -5.64 & -0.33 & 20.23 & 21.30 & 2461.1760 & 0.0267 \\
\hline $5663-7$ & $19: 44: 37.32$ & $-14: 56: 03.0$ & 0.10 & $3.89(4)$ & -7.30 & 1.42 & 21.29 & - & 2460.5838 & 0.0778 \\
\hline $10900-6$ & $19: 44: 40.47$ & $-14: 47: 03.9$ & 1.00 & $5.23(10)$ & -3.77 & 0.61 & 16.04 & 17.23 & 2459.7992 & 0.4184 \\
\hline $12194-7$ & $19: 44: 40.48$ & $-14: 51: 05.3$ & 1.96 & $15.95(40)$ & -2.71 & - & 18.85 & 20.73 & 2465.1790 & 0.3190 \\
\hline $5668-7$ & $19: 44: 41.63$ & $-14: 56: 35.6$ & 0.52 & $0.19369(10)$ & -7.97 & 0.73 & 18.88 & 19.00 & 2458.8996 & 0.0029 \\
\hline $6115-7$ & $19: 44: 47.15$ & $-14: 49: 22.6$ & 0.58 & 19(1) & -5.15 & 1.42 & 20.50 & 21.16 & 2459.0513 & 0.3800 \\
\hline $5698-6$ & $19: 44: 48.86$ & $-14: 44: 00.3$ & 0.30 & $2.609(16)$ & -5.13 & -0.34 & 19.79 & 20.87 & 2460.1238 & 0.0522 \\
\hline $12770-6$ & $19: 44: 49.13$ & $-14: 43: 59.1$ & 1.64 & $25.35(1.0) \mathrm{e}$ & -3.51 & 0.20 & 17.18 & 19.76 & 2473.5554 & 1.2675 \\
\hline $8846-7$ & 19:44:51.95 & $-14: 54: 31.1$ & 1.03 & $33(2)$ & -4.21 & 0.62 & 18.78 & 20.38 & 2472.6413 & 0.9900 \\
\hline $6366-5$ & $19: 44: 54.78$ & $-14: 38: 19.2$ & 0.17 & $0.13087(5)$ & -6.82 & 2.12 & 19.43 & - & 2458.8946 & 0.0023 \\
\hline $6123-7$ & $19: 44: 55.03$ & $-14: 50: 18.8$ & 0.30 & $11.5(3)$ & -5.22 & 1.14 & 15.73 & 16.53 & 2460.6128 & 0.2300 \\
\hline $6867-7$ & $19: 44: 55.57$ & $-14: 50: 06.6$ & 0.56 & $8.49(11)$ or $0.5296(5)$ & -4.58 & - & 18.93 & 19.40 & 2464.7121 & 0.5094 \\
\hline $1-3$ & $19: 44: 57.51$ & $-14: 45: 02.8$ & 0.26 & $0.39669(20)$ & -8.27 & 0.34 & 20.10 & 21.04 & 2459.1251 & 0.0079 \\
\hline $803-3$ & $19: 45: 01.26$ & $-14: 45: 40.0$ & 0.59 & $66(3) \mathrm{e}$ & -5.07 & 0.01 & 17.31 & 19.58 & 2498.7443 & 1.2000 \\
\hline $2536-3$ & $19: 45: 04.18$ & $-14: 45: 03.9$ & 1.20 & $5.10(5)$ & -3.91 & 0.48 & 15.84 & 17.58 & 2459.2154 & 0.1020 \\
\hline $203-3$ & $19: 45: 04.40$ & $-14: 42: 48.6$ & 0.10 & $2.83(1)$ or $1.539(1)$ & -6.69 & 1.81 & 18.15 & 19.92 & 2462.2903 & 0.0840 \\
\hline $77-4$ & $19: 45: 06.75$ & $-14: 37: 07.2$ & 0.03 & $21.3(1.3)$ & -8.17 & 1.28 & 16.64 & 18.40 & 2460.5098 & 0.4260 \\
\hline $2516-1$ & $19: 45: 10.89$ & $-15: 01: 58.1$ & 1.62 & $3.45(1) \mathrm{e}$ & -3.47 & -0.38 & 18.39 & 18.44 & 2459.1073 & 0.0690 \\
\hline $339-1$ & $19: 45: 12.68$ & $-15: 03: 05.5$ & 0.18 & $0.14087(10)$ & -5.86 & 1.31 & 15.42 & 16.64 & 2458.9011 & 0.0014 \\
\hline $197-4$ & $19: 45: 15.61$ & $-14: 34: 59.7$ & 0.13 & $1.556(9)$ & -7.37 & 1.30 & 17.18 & 18.57 & 2461.9783 & 0.3110 \\
\hline $488-3$ & $19: 45: 16.36$ & $-14: 45: 15.9$ & 0.56 & $6.66(3) \mathrm{e}$ & -5.61 & -0.41 & 17.34 & 19.09 & 2462.9829 & 0.0330 \\
\hline $28-4$ & $19: 45: 16.55$ & $-14: 37: 53.8$ & 0.06 & $0.7363(5)$ & -8.59 & 0.76 & 17.00 & 18.30 & 2461.7467 & 0.0221 \\
\hline $244-2$ & $19: 45: 18.29$ & $-14: 50: 19.9$ & 0.65 & $0.2164(1) \mathrm{e}$ & -6.83 & 0.93 & 16.76 & 18.41 & 2459.0444 & 0.0022 \\
\hline $258-2$ & $19: 45: 21.01$ & $-14: 51: 54.5$ & 0.16 & $1.84(1)$ & -6.67 & 1.29 & 17.67 & 19.55 & 2458.8935 & 0.0184 \\
\hline $225-4$ & $19: 45: 25.35$ & $-14: 33: 26.7$ & 0.69 & $0.40204(20) \mathrm{e}$ & -7.00 & 0.84 & 19.57 & 21.04 & 2459.1897 & 0.0040 \\
\hline $5145-1$ & $19: 45: 36.77$ & $-14: 58: 00.5$ & 0.41 & $0.34(1)$ & -6.54 & 1.28 & 16.56 & 18.08 & 2459.1386 & 0.0024 \\
\hline $5151-1$ & $19: 45: 38.02$ & $-14: 59: 50.1$ & 0.39 & $10.75(40)$ & -7.24 & 1.18 & 18.77 & 19.48 & 2463.9651 & 0.1075 \\
\hline $5296-1$ & $19: 45: 40.97$ & $-15: 00: 03.1$ & 0.12 & $9.18(25)$ & -6.33 & 1.42 & 17.13 & 18.46 & 2466.0132 & 0.0918 \\
\hline $6232-1$ & $19: 45: 56.02$ & $-15: 00: 21.5$ & 1.03 & $0.13359(3) \mathrm{e}$ & -4.44 & 1.01 & 15.01 & 16.33 & 2458.9510 & 0.0013 \\
\hline $16881-3$ & $19: 45: 57.08$ & $-14: 44: 13.3$ & 0.25 & $0.16566(5) \mathrm{e}$ & -7.45 & 1.06 & 19.90 & 20.84 & 2460.8813 & 0.0033 \\
\hline $16981-2$ & $19: 45: 58.85$ & $-14: 51: 49.2$ & 0.32 & $9.18(20)$ & -5.23 & 0.25 & 17.44 & 18.30 & 2466.5456 & 0.2754 \\
\hline $17011-3$ & $19: 45: 59.21$ & $-14: 43: 08.8$ & 0.17 & $15.7(6)$ & -6.88 & 0.87 & 17.47 & 19.21 & 2460.8582 & 0.1260 \\
\hline $4584-4$ & $19: 46: 03.38$ & $-14: 31: 46.2$ & 0.08 & $5.59(3)$ & -8.9 & 0.86 & 20.53 & 20.62 & 2463.4753 & 0.0838 \\
\hline
\end{tabular}


Table 2. Candidates for periodically variable stars towards NGC 6822. Nomenclature is as in Table 1. This is an excerpt, the whole table is available in electronic form at the CDS.

\begin{tabular}{ccccccccc}
\hline \hline ID & alpha(2000) & $\operatorname{Dec}(2000)$ & $T$ (days) & $\Delta I(\mathrm{mag})$ & $M_{I}$ & $(V-I)_{0}$ & $I$ & $V$ \\
\hline $449-8$ & $19: 43: 56.76$ & $-15: 04: 10.5$ & 178 & 1.34 & -4.52 & 1.67 & 19.48 & 21.62 \\
$1135-8$ & $19: 43: 58.54$ & $-14: 59: 25.8$ & 147 & 1.09 & -4.08 & - & 19.92 & - \\
$662-8$ & $19: 44: 05.08$ & $-14: 57: 37.4$ & 94 & 0.51 & -4.90 & 2.07 & 19.10 & 21.63 \\
$664-8$ & $19: 44: 05.28$ & $-15: 02: 43.0$ & 120 & 0.36 & -5.47 & 1.67 & 18.54 & 20.67 \\
$919-5$ & $19: 44: 09.79$ & $-14: 36: 26.9$ & 170 & 0.64 & -4.46 & 1.89 & 19.54 & 21.89 \\
$757-6$ & $19: 44: 15.51$ & $-14: 44: 10.4$ & 120 & 0.53 & -4.77 & 2.12 & 19.24 & 21.82 \\
$828-7$ & $19: 44: 15.96$ & $-14: 50: 41.2$ & 182 & 0.65 & -4.77 & 2.08 & 19.24 & 21.78 \\
$482-7$ & $19: 44: 19.43$ & $-14: 51: 31.7$ & 109 & 0.34 & -5.05 & 2.09 & 18.95 & 21.50 \\
$5713-8$ & $19: 44: 23.14$ & $-15: 02: 27.5$ & 144 & 0.37 & -4.74 & 2.09 & 19.27 & 21.82 \\
$6246-7$ & $19: 44: 28.59$ & $-14: 50: 44.6$ & 141 & 0.72 & -4.65 & 1.97 & 19.36 & 21.79 \\
\hline
\end{tabular}

Table 3. "Non-periodic" variable stars towards NGC 6822. This is an excerpt, the whole table is available in electronic form at the CDS. The identification number of the star, the field number, coordinates, $I$-band amplitude, absolute $I$ magnitude and dereddened $(V-I)_{0}$ colour are given, as well as the $I$ and $V$ magnitudes. $S / N$ is the ratio between the amplitude of the highest peak in the periodogram and the noise amplitude. Note: many of these variables may turn out to be periodic once the time baseline of the observations will be extended.

\begin{tabular}{cccccccccc}
\hline \hline ID & Field & alpha(2000) & $\operatorname{Dec}(2000)$ & $\Delta I(\mathrm{mag})$ & $M_{I}$ & $(V-I)_{0}$ & $I$ & $V$ & $S / N$ \\
\hline 17 & 1 & $19: 45: 13.32$ & $-14: 58: 44.6$ & 0.03 & -8.29 & 1.51 & 15.71 & 17.69 & 8.03 \\
554 & 1 & $19: 45: 07.45$ & $-14: 59: 23.6$ & 0.09 & -5.06 & 2.42 & 18.94 & 21.83 & 6.56 \\
564 & 1 & $19: 45: 08.15$ & $-15: 02: 16.1$ & 0.05 & -4.66 & 1.94 & 19.34 & 21.75 & 4.45 \\
572 & 1 & $19: 45: 08.99$ & $-14: 57: 46.1$ & 0.11 & -4.76 & - & 19.25 & - & 6.09 \\
628 & 1 & $19: 45: 14.76$ & $-14: 58: 23.8$ & 0.11 & -4.81 & 2.47 & 19.20 & 22.13 & 5.79 \\
660 & 1 & $19: 45: 18.22$ & $-14: 59: 52.2$ & 0.09 & -4.75 & 2.28 & 19.26 & 22.00 & 5.24 \\
679 & 1 & $19: 45: 20.45$ & $-14: 59: 38.0$ & 0.18 & -5.01 & - & 18.99 & - & 8.94 \\
744 & 1 & $19: 45: 26.92$ & $-14: 57: 41.6$ & 0.13 & -4.87 & 2.45 & 19.14 & 22.05 & 7.41 \\
798 & 1 & $19: 45: 32.34$ & $-15: 01: 01.6$ & 0.05 & -4.82 & 2.01 & 19.19 & 21.66 & 4.79 \\
892 & 1 & $19: 45: 00.18$ & $-15: 00: 55.2$ & 0.53 & -3.64 & - & 20.37 & - & 8.34 \\
\hline
\end{tabular}
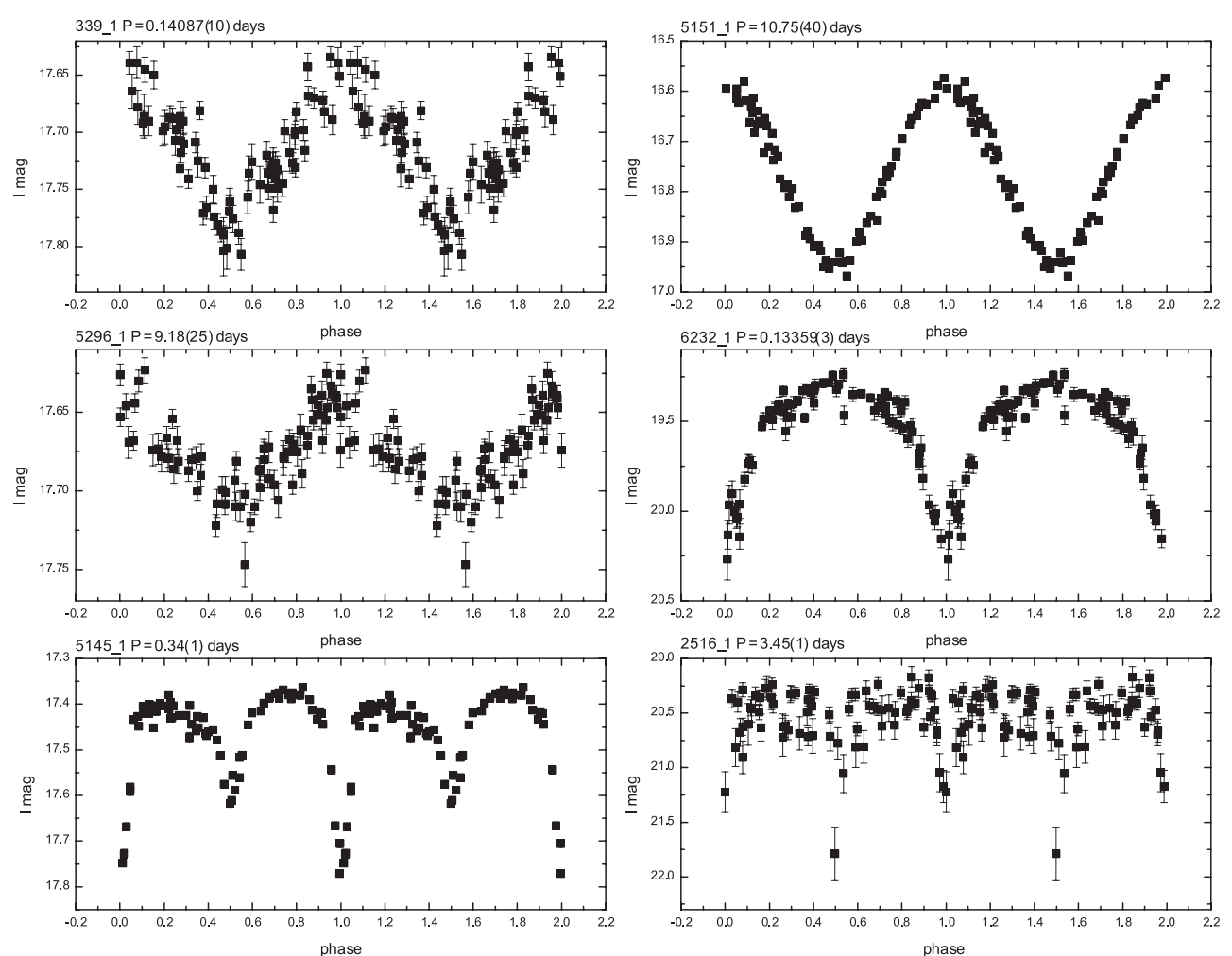

Fig. 1. Examples of light curves for periodic variables. The full atlas is available in the electronic version. 

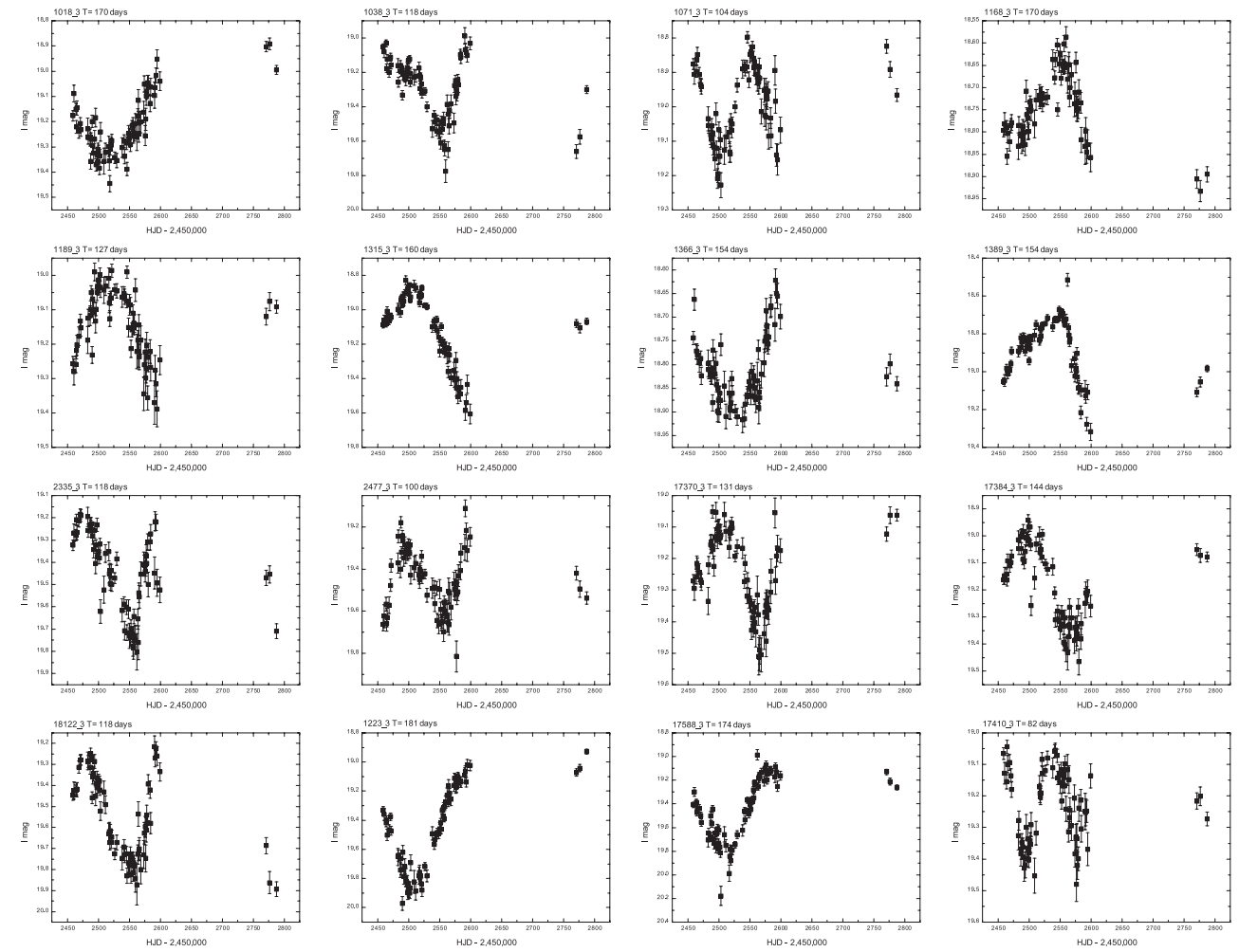

Fig. 2. Examples of light curves for probably periodic variables. The full atlas is available in the electronic version.

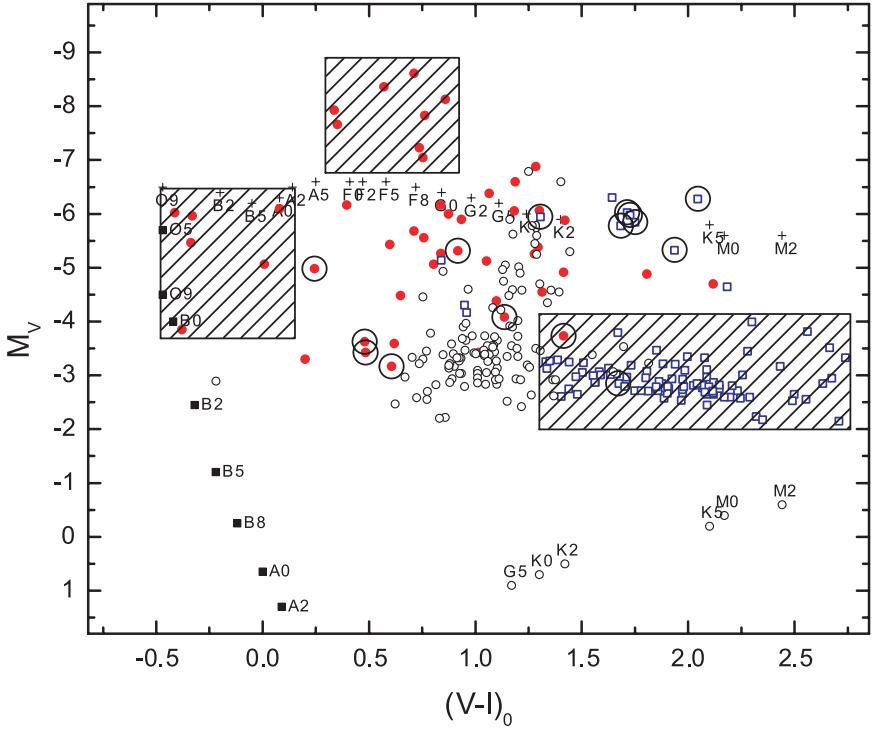

Fig. 3. The colour-magnitude diagram for the variable stars towards NGC 6822. Small filled circles correspond to periodically variable stars, and open squares to candidates for periodic variables. Cepheid candidates are indicated by large circles. The Cepheids reported by P04 are shown as small open circles. The position of the upper blue main sequence is indicated, along with the tracks for giants and supergiants accordingly to Cox (2000). The dashed rectangular boxes include the blue, yellow and red stars discussed in the text.

\subsubsection{Blue variables}

The six stars located in the "blue" part of the CM diagram correspond to three double eclipsing systems (488-3, 803-3 and 2516-1, with periods $6.66,66$ and 3.44 days, respectively) and three periodic stars with periods 0.18 days (377-6), 2.67 days (4907-6), and 2.61 days (5698-6). These later stars show regular "single-wave" light curves with $I$-band amplitude $0.3-0.5 \mathrm{mag}$. The periods could be rotational, except for 377-6, which could be a short-period RR Lyrae star of the galactic halo of type RRc.

\subsubsection{New Cepheids}

We looked for new Cepheids in our sample by constructing $I$ versus $\log P$ diagrams for our periodic stars, and overplotting the Cepheids found by P04. The stars lying close to these Cepheids (9 stars), were selected as new Cepheid candidates (Fig. 4). After examining their light curves for classification consistency (shape, period and amplitude), we concluded that they are indeed likely to be Cepheids, except 2516-1 which is an eclipsing binary. The new Cepheid candidates are labeled 16981-2, 2536-3, 390-6, 1433-6, 10900-6, 6115-7, 6123-7 and 6867-7. We did the same exercise with the quasiperiodic variables with periods in excess of 100 days. We found eight stars lying close to the $P L$ relationship (Fig. 4); they are the variables $210-1,57-2,65-3,6193-5,4641-6,4741-6$, 4898-6 and 449-8. Their long time scale of variability (larger than 110 days) and very small amplitudes (less than $0.2 \mathrm{mag}$ ), apart from their position in the CM diagram ( $V-I$ usually larger than 1.6), pointed to a classification different from Cepheids. However, when plotting these objects on the period-luminosity relationship they turn out (except 449-8, which is clearly an outlier, and has a low luminosity) to be almost aligned with the "bona-fide" Cepheids (Fig. 5). In the $I$-band $P L$ relation, these 


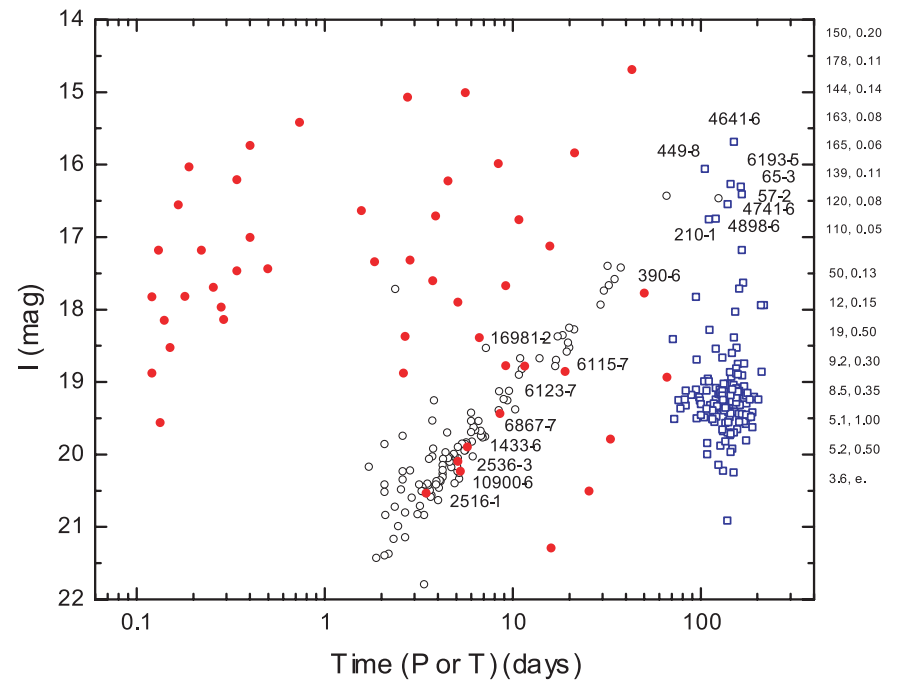

Fig. 4. The "Cepheid-filtering" diagram. We include here true periods (dots) for periodic stars, and time scales of variability (squares) for probably periodic stars. The numbers at the right side correspond to the pairs [period (days), amplitude] for the Cepheid candidates. Cepheids found by P04 are shown as open circles. The new Cepheid candidates are labeled. Note the bright long-period candidates. They were not flagged as possible Cepheids in P04 due to their very small amplitudes.

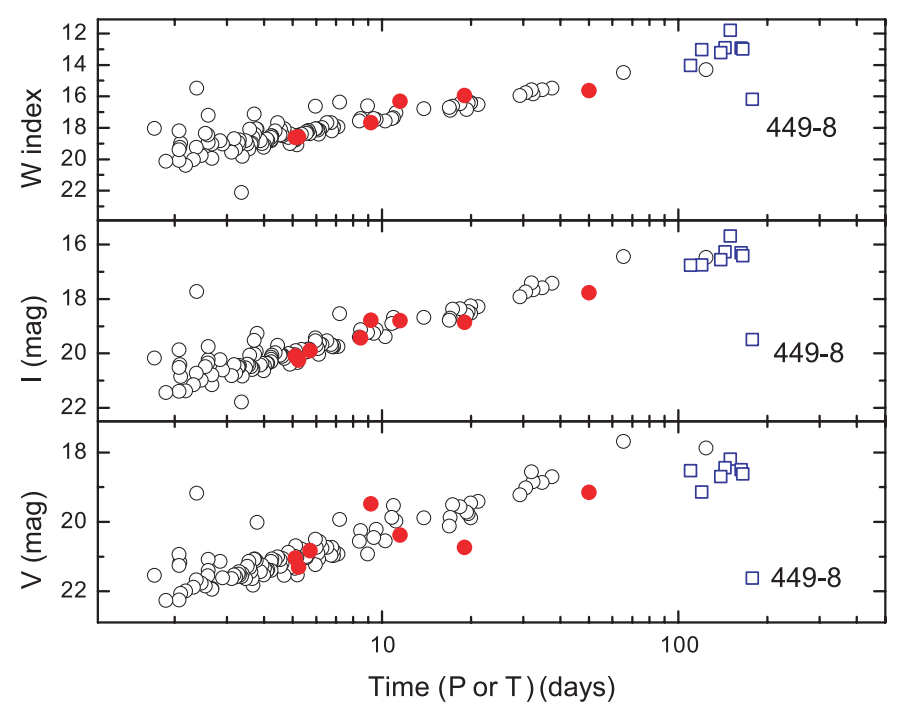

Fig. 5. The $P L$ relationships (using the period, or time scale of variability) for the Cepheids of Pietrzynski et al. (2004, open circles), our periodic Cepheid candidates (filled circles) and our long-period, small-amplitude Cepheid candidates (open squares). These diagrams show that, except for $449-8$, the long-period Cepheid candidates closely follow the $P L$ relationships in the I and reddening-free $W$ bands, but are underluminous in the $V$ band.

7 stars almost follow the $P L$ sequence defined by the shorter period stars, but are somewhat underluminous (as compared to the expected $I$ magnitudes when extrapolating the shorterperiod sequence). In the $V$-band $P L$ relation ( $V$ vs. $\log P$ ), the effect is much stronger; the 7 stars are at least 1 mag fainter than expected if they were normal Cepheids, assuming that the Cepheid PL relation keeps being linear (in the magnitude vs. $\log P$ plot) up to such high periods. However, in the reddening-free Wesenheit magnitude these 7 objects lie exactly on the extension of the $P L$ relation defined by all the normal, shorter-period Cepheids (Fig. 5). In the color-magnitude diagram, the seven stars all seem to be too red to lie within the Cepheid instability strip (Fig. 3). However, we caution here that the red edge of the instability strip is currently not well defined; models have still trouble to incorporate convection correctly into the models. Therefore, it seems not completely out of the question that these objects are very red Cepheids, very close to the red edge of the instability strip, and perhaps pushed slightly beyond it by excessive differential reddening. This would be consistent with their very low amplitudes. In the period-color diagram $\left((V-I)_{0}\right.$ vs. $\left.\log P\right)$, the 7 stars are by $0.5-$ 1.0 mag redder than what would be expected from the prolongation of the period-color relation defined by the shorter-period Cepheids. This might argue, in principle, against a classification as Cepheids; however, the intrinsic dispersion of Cepheid period-colour relations is large.

As a conclusion at this point, things are a bit confusing; the nature of these stars is not really clear. While they could be Galactic foreground stars (but 7 being so similar in their characteristics is hard to imagine), or variable red supergiants, they could also be very long-period Cepheids. There is very little known empirically and theoretically about such stars, and their properties. In the literature, it has been claimed that Cepheids with $P>100$ days have a tendency of departing from the $P L$ sequence of the shorter-period Cepheids, towards lower luminosities, exactly what we see. On the other hand, the Cepheid classification is consistent with the low metallicity of NGC 6822 (e.g. Clementini et al. 2003) and the theoretical expectation that the longest period Cepheids should be found in environments of low metallicity (Aikawa \& Antonello 2000). If these seven objects are very long-period Cepheids, they must be very massive, and therefore, very young stars; they could then perhaps still be dust-enshrouded to some extent, which could partly explain why these stars are so red. It will be very interesting and important to follow up on these variables because they might give us a clue to find out if such extremely long-period Cepheids indeed exist, and if so, if they follow the $P L$ relation defined by the shorter-period (less than 100 days) Cepheids. This is a very important issue for the use of Cepheids as distance indicators, where the longest-period and brightest Cepheids are potentially the most useful ones. Little is known about such possible Cepheids of extremely long periods because of their extreme paucity, and NGC 6822 could be a key galaxy to study them, if the Cepheid nature of these seven enigmatic objects is confirmed by future observations.

\subsubsection{Stars in the yellow supergiant region}

Eight stars are located above the yellow supergiant track in Fig. 3. We investigated if these stars could be related to the yellow hypergiant stars reviewed by de Jagger (1998). Yellow hypergiants are possibly evolved stars evolving from the red supergiant phase to the blue phase. The candidate stars are 1-3, $28-4,4584-4,32-5,58-5,6066-5,6140-5$ and 5668-7. By examination of the light curves of these objects, we find that 
$6140-5$ is a near-contact binary with orbital period 0.34 days. For this period, the components of a contact binary should be much less luminous, so we conclude that this is a foreground object that does not belong to NGC 6822. The other objects have periods 0.4 days $(1-3, A=0.26), 2.8$ days (28-4, $A=0.06), 5.6$ days (4584-4, $A=0.08-0.05), 43$ days (32-5, $A=0.05), 2.8$ days $(58-5, A=0.10), 8.3$ days $(6066-5$, $A=0.31$ ) and 0.19 days $(5668-7, A=0.52)$. As we can see, the periods are rather short, much shorter than those observed in pulsating yellow hypergiants (hundreds of days). On the other hand, the periods are also too short to allow a companion star around a supergiant, so we conclude that these stars are very likely members of our galaxy. Stars 1-3 and 5668-7 could be RR Lyrae stars in the halo of our galaxy.

\subsubsection{The long period variables}

The last group in our discussion corresponds to the red variables in the right rectangle in Fig. 3. These stars have $1.3<$ $(V-I)_{0}<2.8$ and $-4.1<M_{V}<-2.0$. They are slow variables, with time scales of variability between 70 and 190 days. The upper limit is due to our selection mechanism; the application of the automatic period-searching algorithm hardly finds stars with longer periodicities due to the time baseline defined by our data. Interestingly, some of these stars show also variability on shorter time scales (see Fig. 2). Their $I$ amplitudes show a smooth distribution peaking at $0.5 \mathrm{mag}$ (Fig. 6). These stars are likely Mira stars, or semiregular variables (Kholopov et al. 1985). There is no correlation between time scale of variability and colour, amplitude or magnitude for these stars. Our stars occupy a region of higher luminosity than the tip of the red giant branch (TRGB) and we do not see $P L$ relationships for them as those observed in the Magellanic Clouds for TRGB stars (Fraser et al. 2005). However, we note that bright stars do not show large amplitude variability (Fig. 6). This could be explained if bright stars showing large amplitudes also show predominantly longer periods, and therefore would escape from detection in our present study.

\subsection{The non-periodic variable stars}

Figure 7 shows the CM diagram for the 1019 non-periodic variable stars. We observe some stars close to the main sequence, in the region of high-mass stars. We also find many high luminosity stars in the region of the supergiants of types G0-M2. $I$-band amplitudes for these stars vary from 0.02 to $1.18 \mathrm{mag}$, with a mean of $0.15( \pm 0.14 \mathrm{std}) \mathrm{mag}$, and no correlation with $(V-I)_{0}$ colour. The most notable feature of the diagram is the strong concentration in the region of the red giants. For these red stars we show the histogram of the $I$-amplitudes in Fig. 8, along with their distribution with absolute $I$ magnitude. The difference with Fig. 6 is notable. We observe smaller amplitudes and no correlation with luminosity, which confirms our suggestion that the deficit of stars with large amplitudes and high luminosities observed in Fig. 6 is an artifact. Another point of interest is the large number of stars in the region occupied by the classical Cepheids discovered by P04. It is hard to
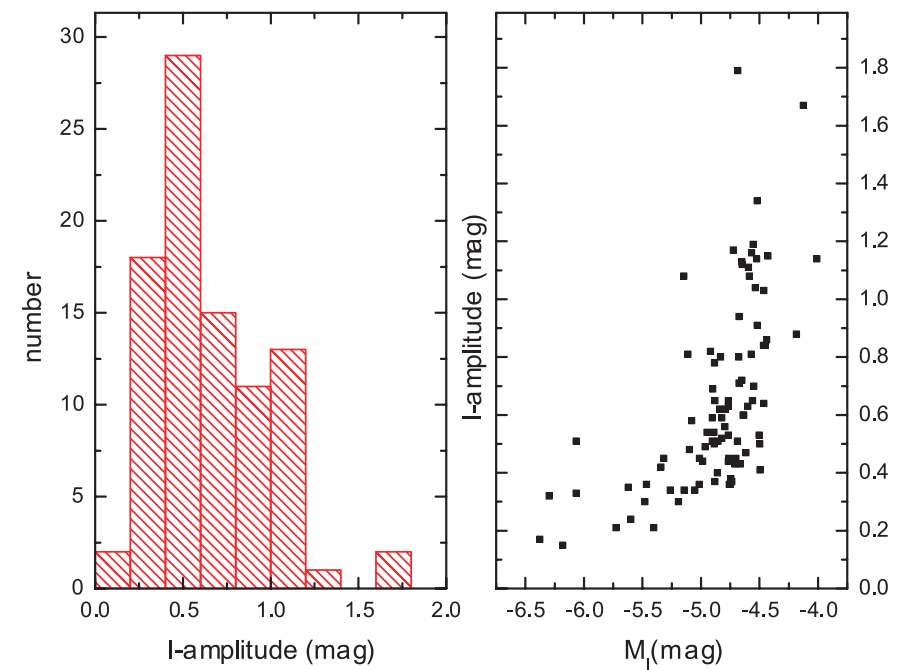

Fig. 6. The left panel shows the histogram of the amplitudes for the variables in the bottom-right rectangle in Fig. 3. The right panel shows the amplitude as a function of the absolute $I$-band magnitude.

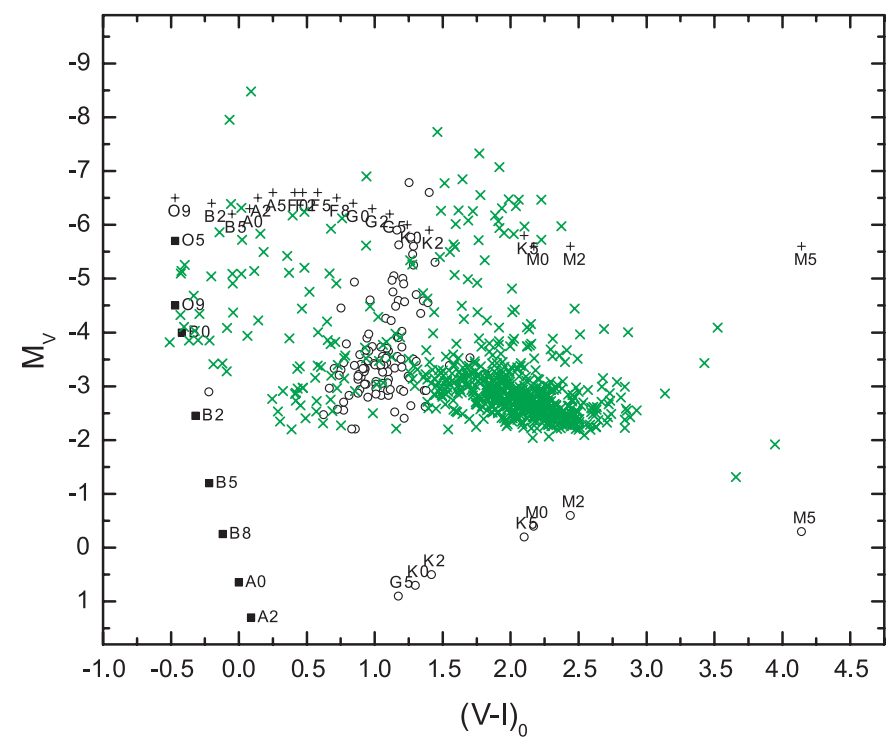

Fig. 7. Same as Fig. 3, but for "non-periodic" variable stars (crosses).

imagine that all these stars are foreground galactic stars (see also discussion in Sect. 5), so it is probable, as we already noted in Sect. 4.2, that other kinds of variables are located in the Cepheid instability strip. Spectroscopy is needed to establish the nature of these low-amplitude objects.

\section{Conclusions}

In this paper we have continued our search for extragalactic variable stars making use of the image databases provided by the Araucaria Project (for variable stars detected in NGC 300 in the framework of this project, see Mennickent et al.2004; for details on the Araucaria Project, see for instance Pietrzynski et al. 2002, and more recently Gieren et al. 2005). With this study, we have significantly contributed to the census and understanding of the population of bright variable stars towards the galaxy NGC 6822. We have presented data 

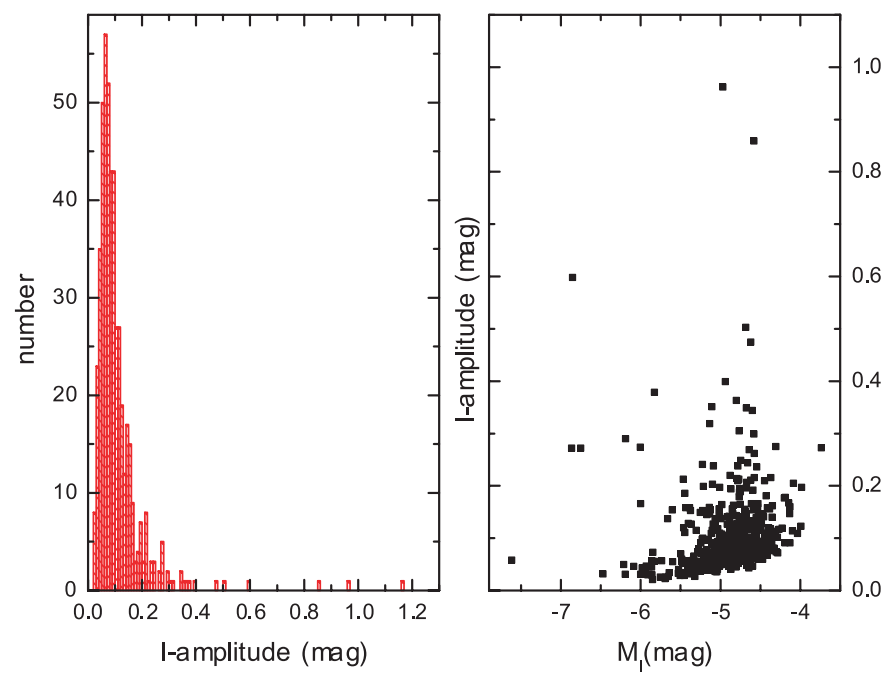

Fig. 8. The left panel shows the histogram of the amplitudes for the red non-periodic variables in Fig. 7 (those with $M_{V}>-4.1$ and $(V-I)_{0}>$ 1.3). The right panel shows the amplitudes plotted against the absolute I-band magnitudes for these stars.

for more than 1200 variable stars, far exceeding the number of previously known objects of this kind toward this galaxy. Unfortunately, for many objects we cannot unambiguously determine their membership to NGC 6822, since contamination by foreground Milky Way stars is a strong problem in such surveys in Local Group galaxies. Our present survey is not an exception, especially due to the low galactic latitude of NGC 6822 $\left(b^{\mathrm{II}}=-18^{\circ}\right)$. According to the Bahcall \& Soneira galaxy model for star densities, we should expect 16.2 foreground stars per square arcminute with magnitudes in the range of $13<V<23$ towards NGC 6822 (Ratnatunga \& Bahcall 1985). This yields approximately $18 \%$ of contamination for the total number of stars detected in our survey. If we translate this figure to the detected variable stars, we would expect that 219 stars of our 1215 objects could be galactic foreground stars. True membership to NGC 6822 can only be established with spectroscopic follow-up surveys and, in some cases, with multicolor photometry (Massey 1998). In any case, our work has been exploratory; the detection of variable star candidates constitutes the first step towards a complete understanding of the variable star population in this galaxy. Our search was limited to $M_{V}<-2$, excluding pulsators like RR-Lyrae and $\delta$ Scuti stars, but we should be able to detect $\beta$ Cephei, Mira and semiregular variables, as well as RV Tau stars. Many of our targets could finally be classified as these kind of variables once follow-up observations become available. Further spectroscopic work and continuous photometric monitoring are therefore encouraged to understand the nature of these variables, and to compare their properties with those of the corresponding populations in other galaxies.
An interesting byproduct of our investigation has been the discovery of a small population of stars which could be strongly reddened, small-amplitude Cepheids of extremely long periods. They seem to follow the Cepheid $P L$ relationship, but turn to be underluminous in the $V$ band. We suspect that these 7 objects might be indeed very young Cepheids which are still embedded in dusty envelopes. Further work will be necessary to corroborate this hypothesis.

Acknowledgements. We acknowledge the referee, Dr. Raffaele Gratton, for useful comments on the first version of this manuscript. R.E.M. acknowledges support by Grant Fondecyt, project number 1030707. W.G., G.P. and R.E.M. acknowledge financial support for this work from the Chilean Center for Astrophysics FONDAP, project number 15010003. This work is based on data obtained with the Warsaw telescope on Las Campanas. We gratefully acknowledge help of some of the members of the OGLE team in the acqisition of these data.

\section{References}

Aerts, C., \& Kolenberg, K. 2005, A\&A, 431, 615

Aikawa, T., \& Antonello, E. 2000, A\&A, 363, 601

Antonello, E., Fugazza, D., Mantegazza, L., Stefanon, M., \& Covino, S. 2002a, A\&A, 386, 860

Antonello, E., Fugazza, D., Mantegazza, L., \& Stefanon, M. 2002b, Inf. Bull. Var. Stars, 5254, 1

Baldacci, L., Matonti, F., Rizzi, L., et al. 2004a, MmSAI, 75, 126

Baldacci, L., Rizzi, L., Clementini, G., \& Held, E. V. 2004b, ASPC, 310, 91

Baldacci, L., Rizzi, L., Clementini, G., \& Held, E. V. 2005, A\&A, 431, 1189

Breger, M., et al. 1993, A\&A, 271, 482

Clementini, G., Held, E. V., Baldacci, L., \& Rizzi, L. 2003, ApJ, 588, L85

Cox A. N. 2000, Allen's Astrophysical Quantities (New York: Springer-Verlag)

de Jager, C. 1998, A\&AR, 8, 145

Fraser, O. J., Hawley, S. L., Cook, K. H., \& Keller, S. C. 2005, AJ, 129, 768

Gieren, W., Pietrzynski, G., Bresolin, F., et al. 2005, The ESO Messenger, 121, 23

Jones, J. H., Christian, C. A., Crowe, R., et al. 1987, BAAS, 19, 1052

Khopolov, P. N., et al. 1985-1988, General Catalogue of Variable Stars, 4th ed. (Moscow: Nauka) (GCVS)

Massey, P. 1998, ApJ, 501, 153

McGonegal, R., McLaren, R. A., Welch, D. L., Madore, B. F., \& McAlary, C. W. 1983, ApJ, 273, 539

McGonegal, R., McLaren, R. A., Welch, D. L., Madore, B. F., \& McAlary, C. W. 1983, ApJ, 273, 539

Mennickent, R. E., Pietrzyński, G., \& Gieren, W. 2004, MNRAS, 350, 679

Pietrzyński, G., Gieren, W., Fouqué, P., \& Pont, F. 2002, AJ, 123, 789

Pietrzyński, G., Gieren, W., Udalski, A., et al. 2004, AJ, 128, 2815

Ratnatunga, K. U., \& Bahcall, J. N. 1985, ApJS, 59, 63

Schlegel, D. J., Finkbeiner, D. P., \& Davis, M. 1998, ApJ, 500, 525

Schmidt, E. G., \& Spear, G. G. 1987, BAAS, 19, 1036

Schwarzenberg-Czerny, A. 1989, MNRAS, 241, 153 


\section{Online Material}


R. E. Mennickent et al.: Bright variable stars in NGC 6822, Online Material p 2
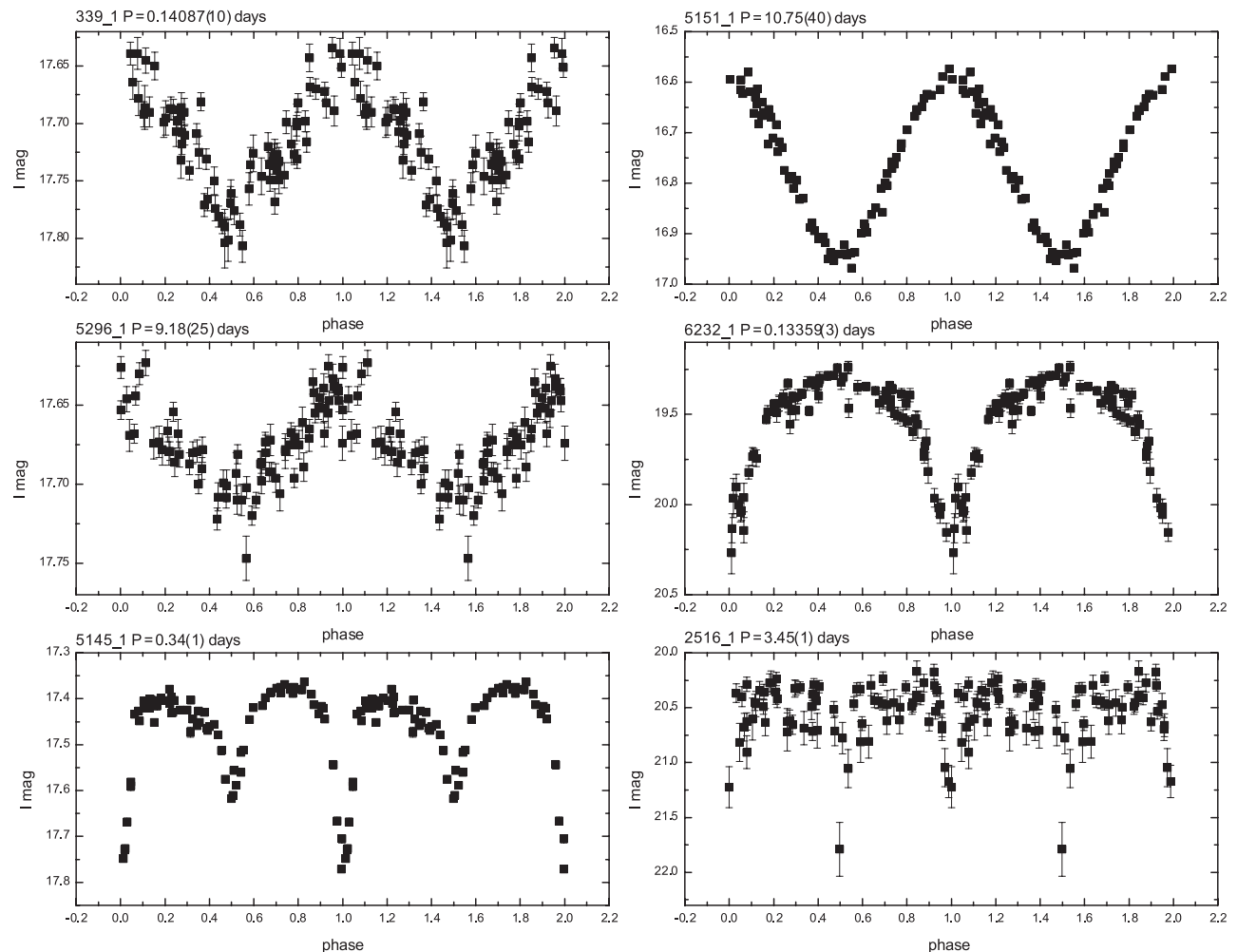

Fig. 1. Light curves of periodic variables.
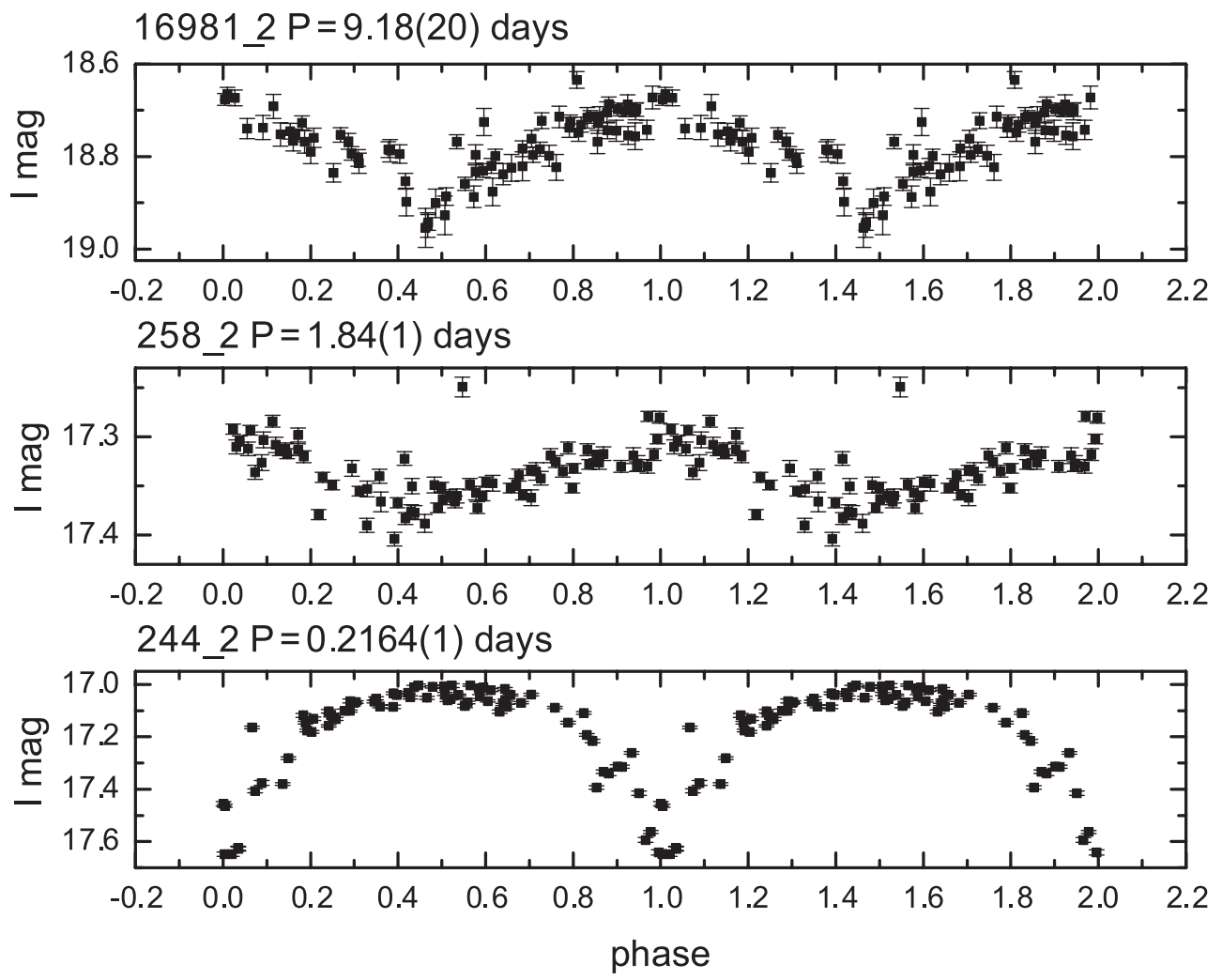

Fig. 1. continued. 
R. E. Mennickent et al.: Bright variable stars in NGC 6822, Online Material p 3
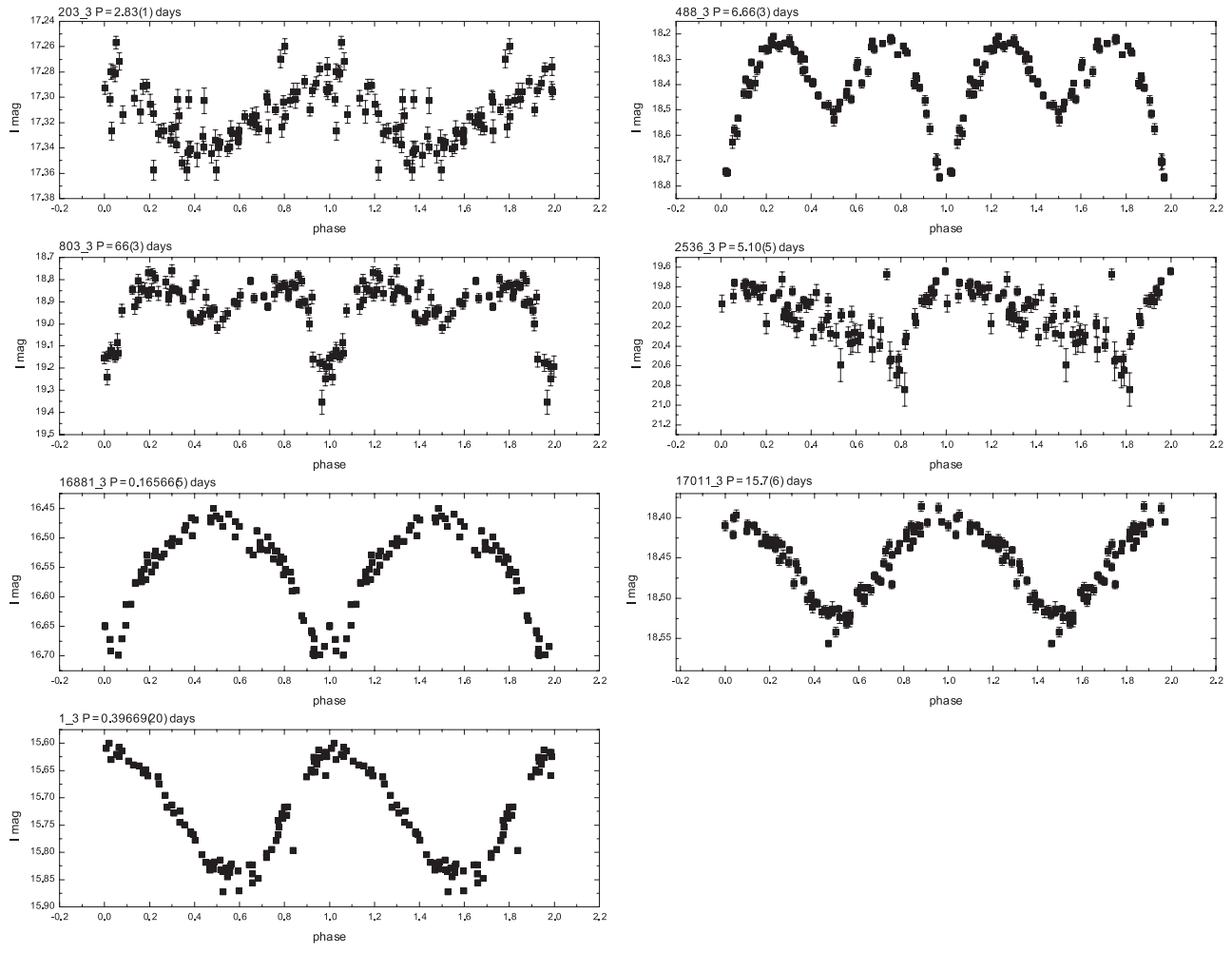

Fig. 1. continued.
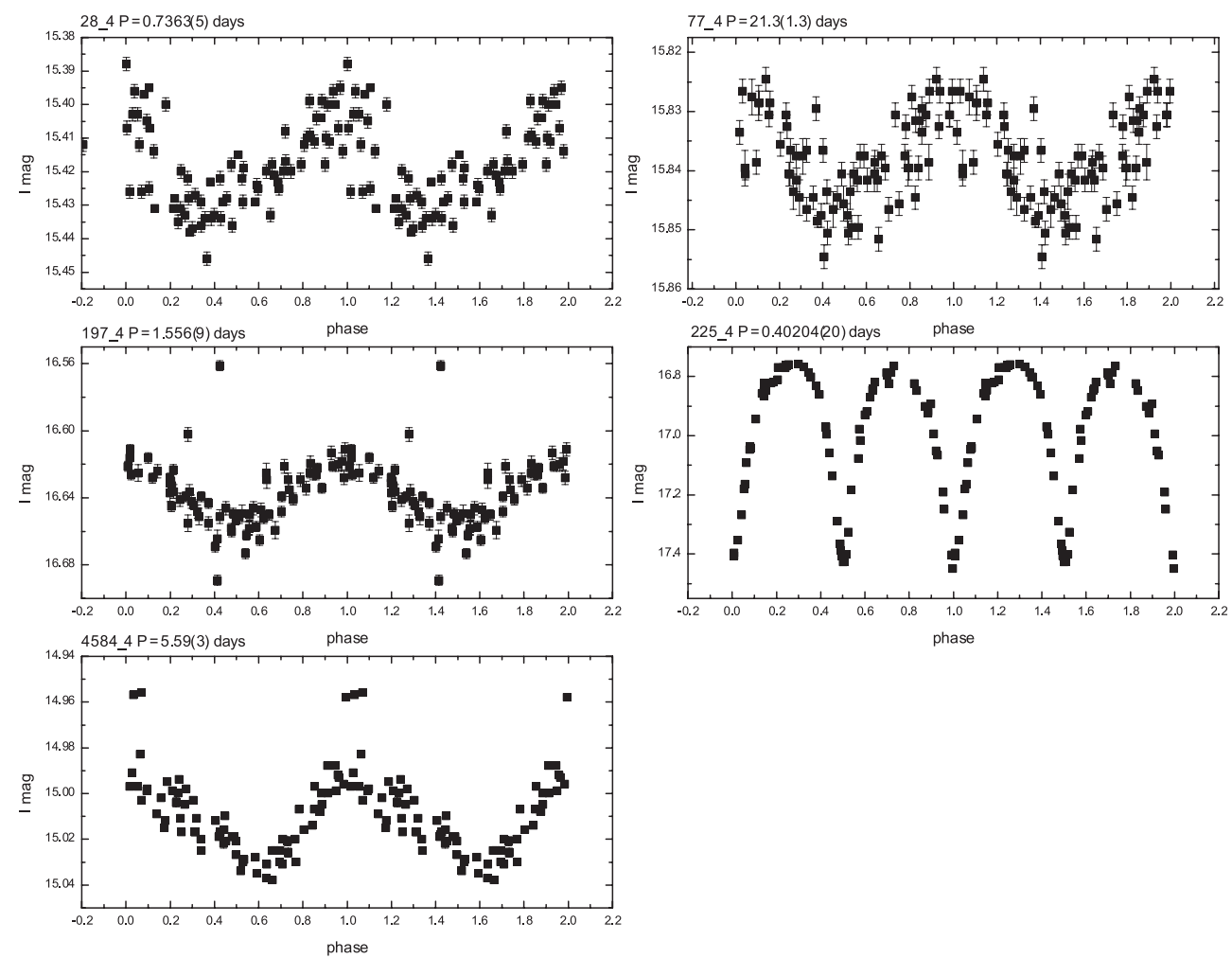

Fig. 1. continued. 
R. E. Mennickent et al.: Bright variable stars in NGC 6822, Online Material p 4
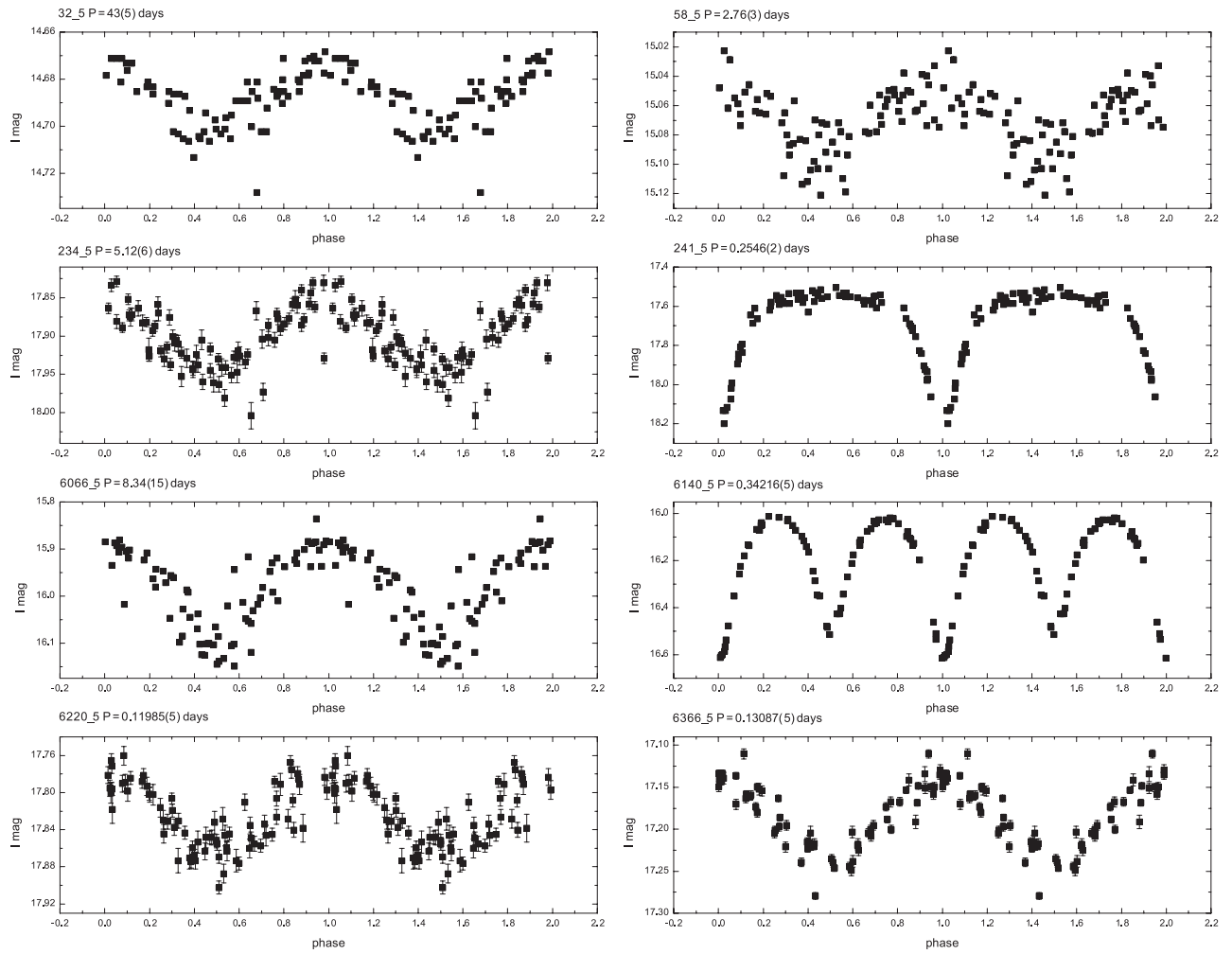

Fig. 1. continued.
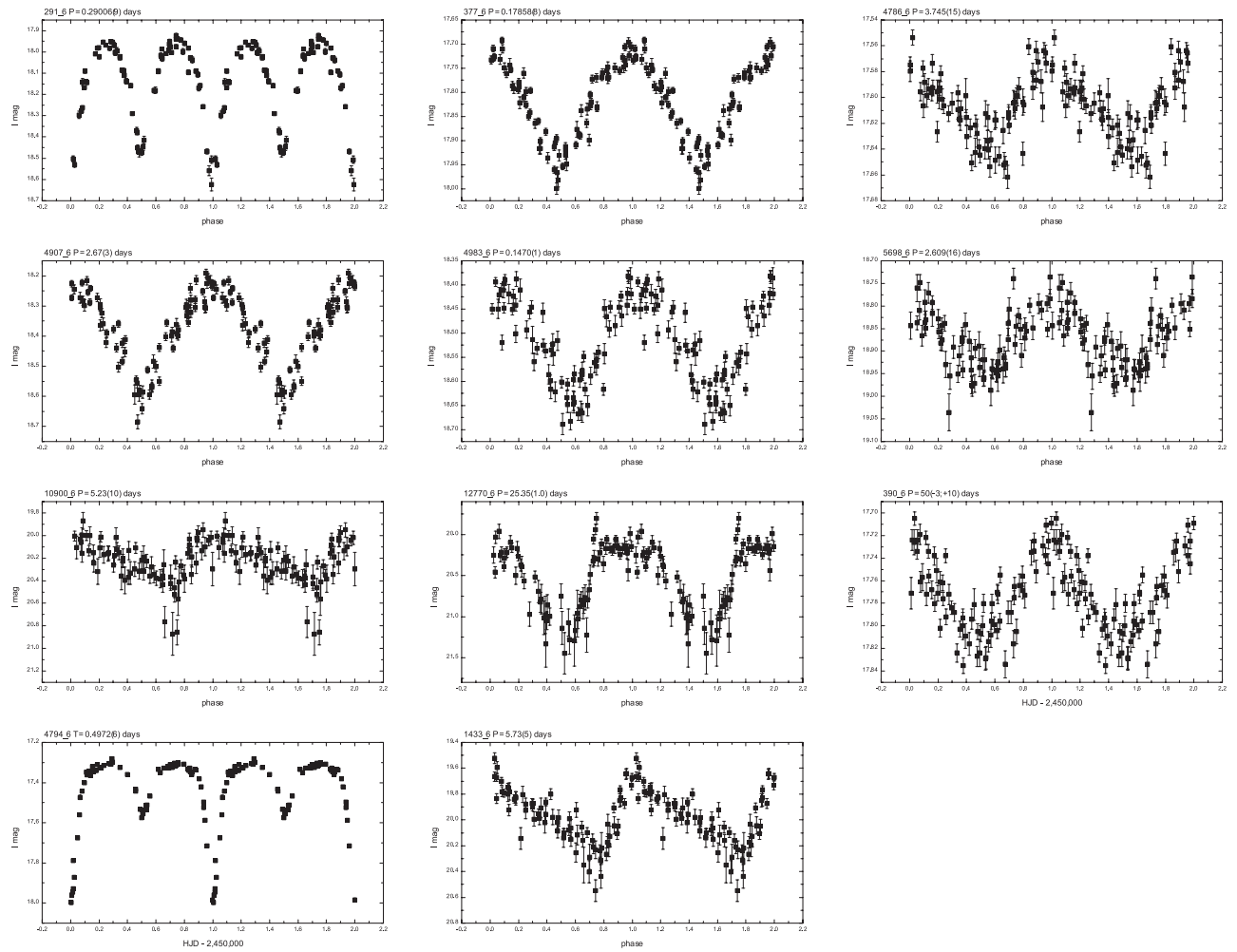

Fig. 1. continued. 
R. E. Mennickent et al.: Bright variable stars in NGC 6822, Online Material p 5
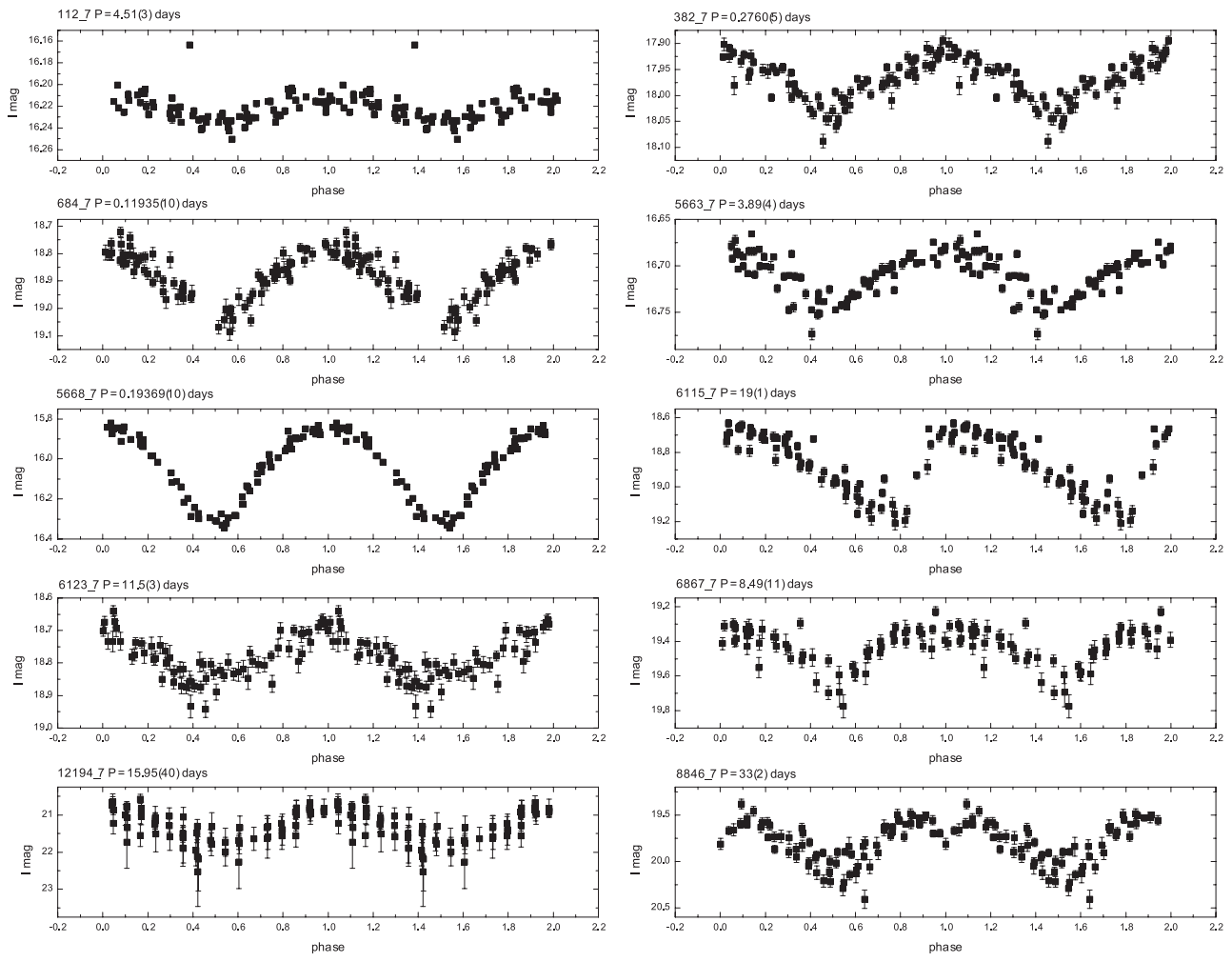

Fig. 1. continued.
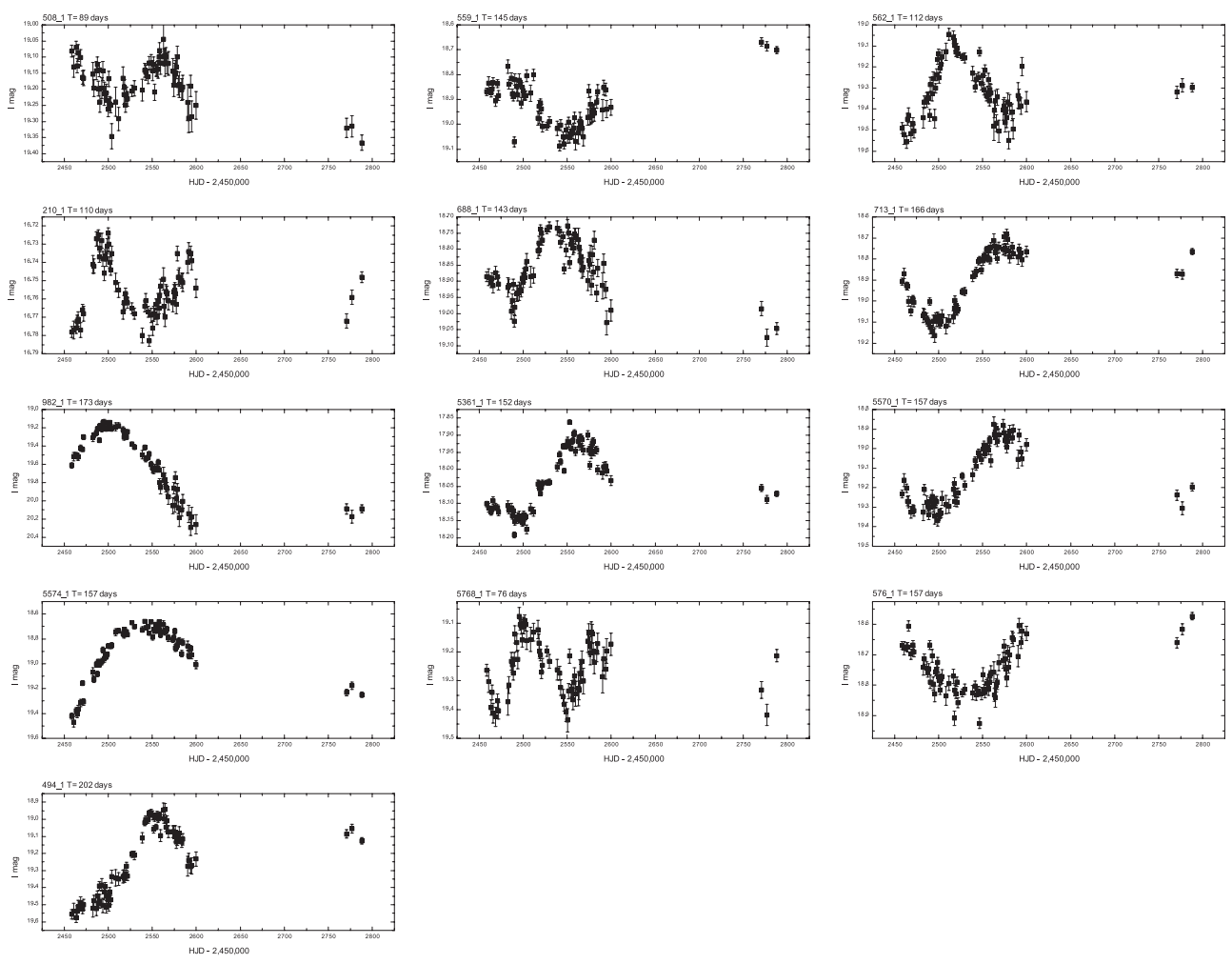

Fig. 2. Light curves of probable periodic variables. 
R. E. Mennickent et al.: Bright variable stars in NGC 6822, Online Material p 6
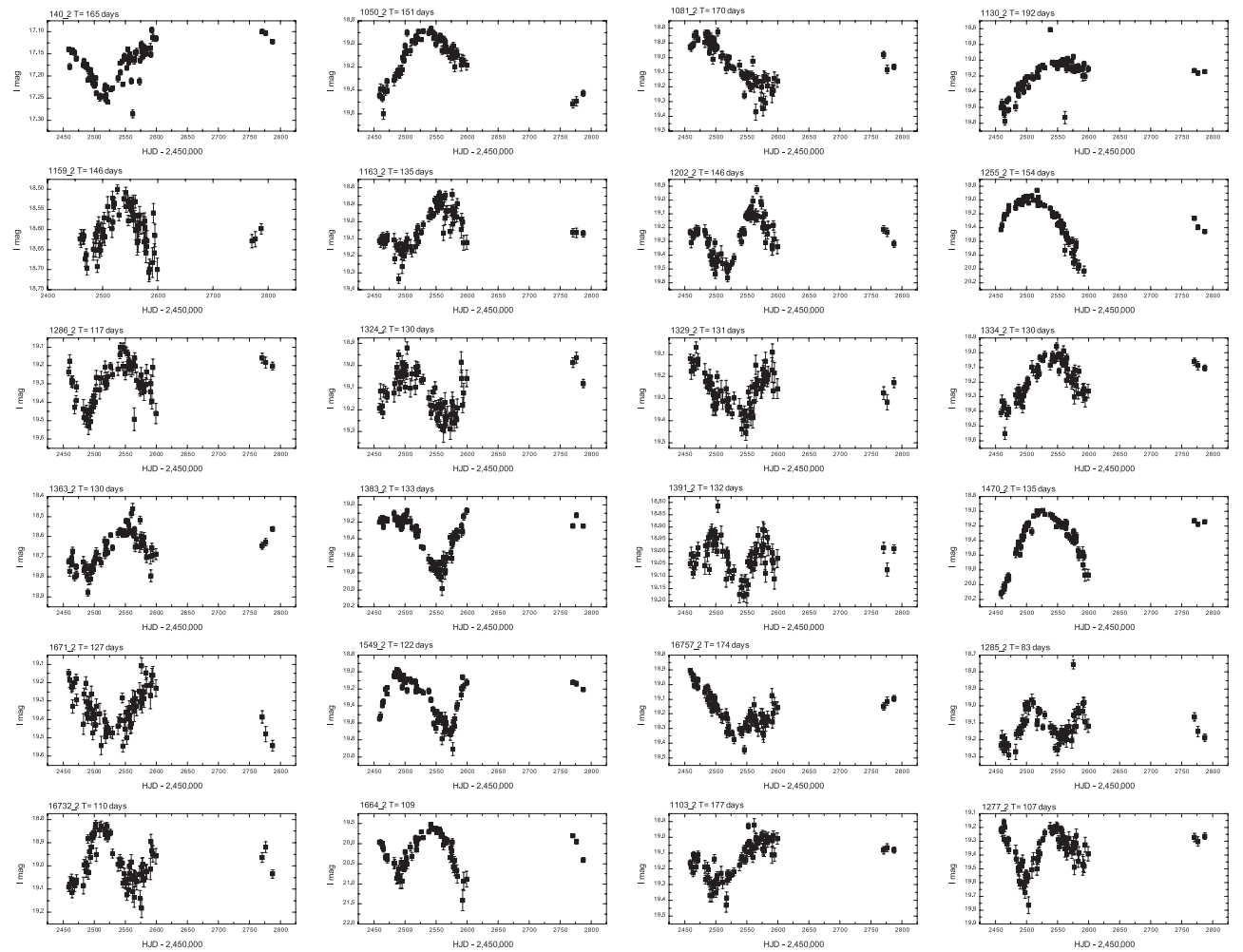

Fig. 2. continued.
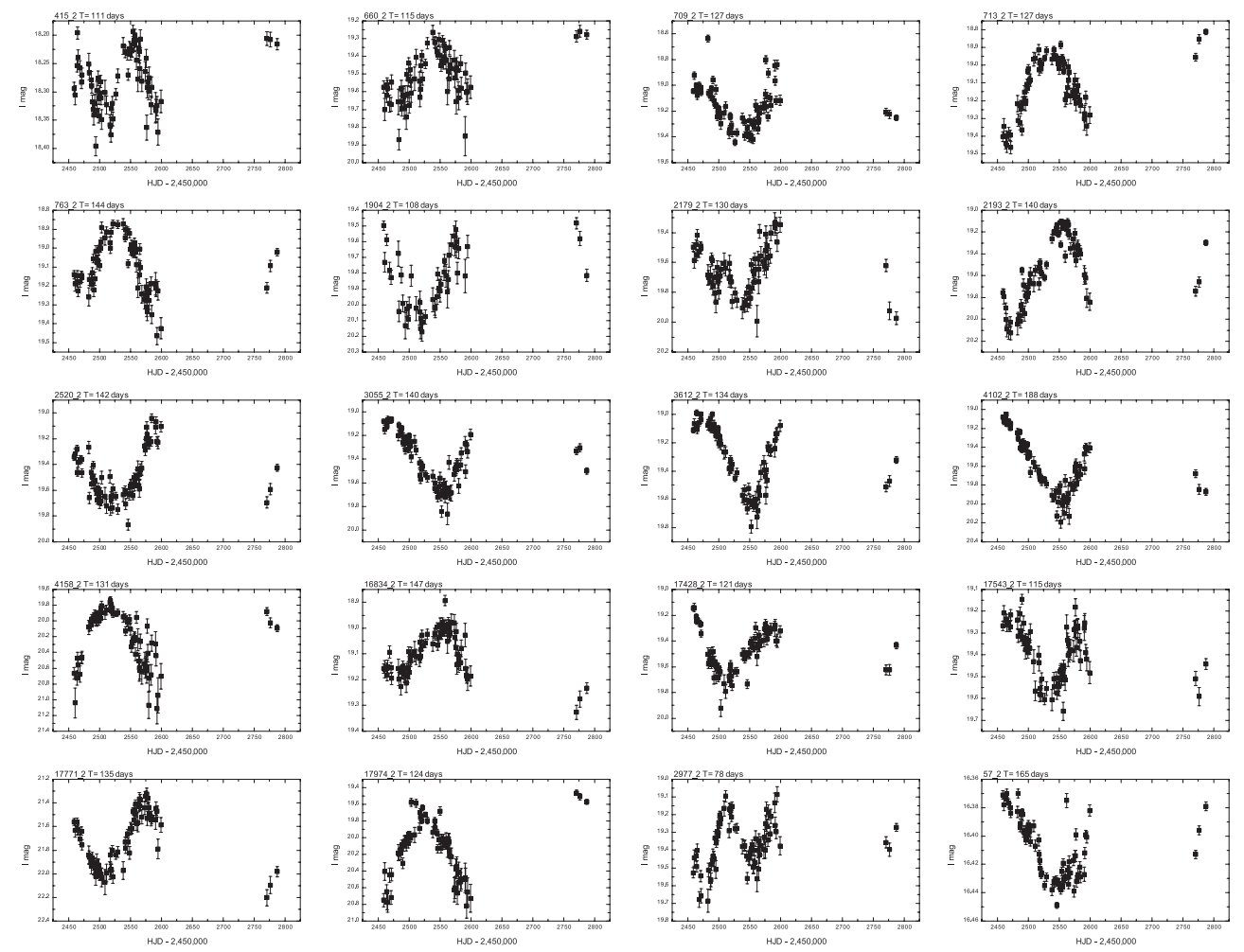

Fig. 2. continued. 
R. E. Mennickent et al.: Bright variable stars in NGC 6822, Online Material p 7
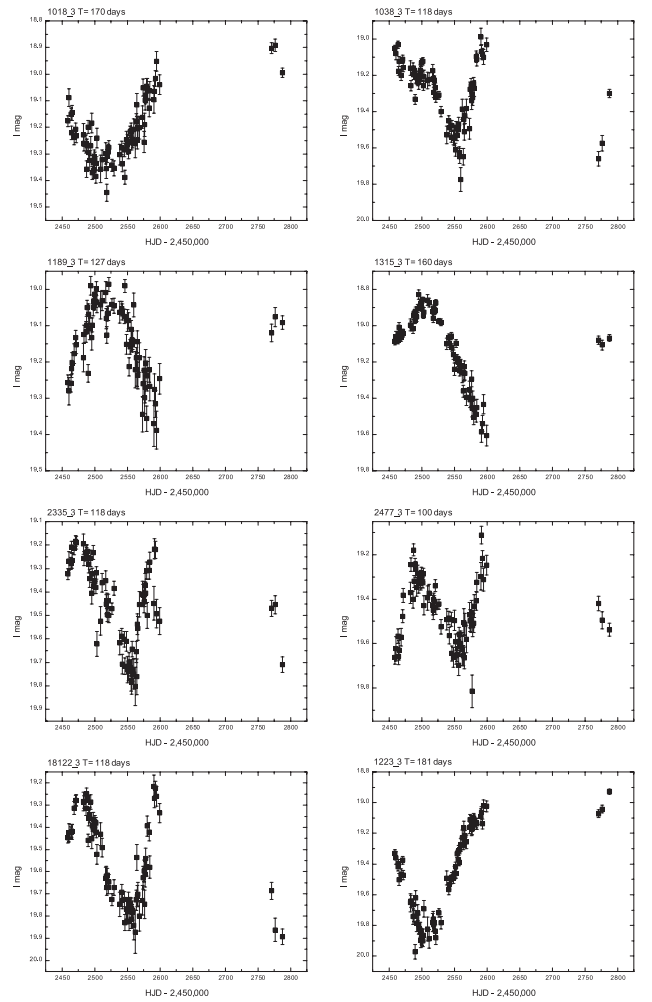

Fig. 2. continued.
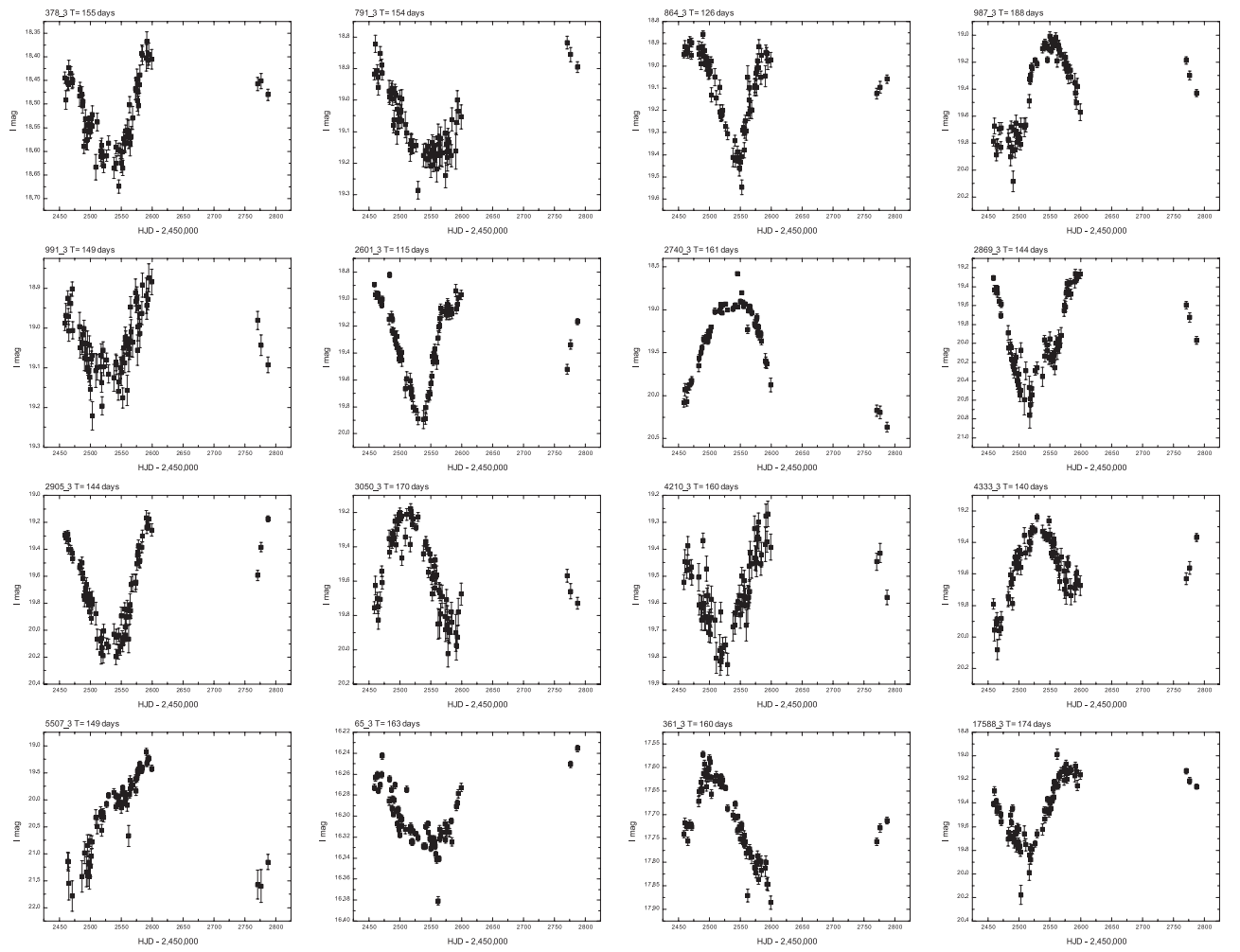

Fig. 2. continued. 
R. E. Mennickent et al.: Bright variable stars in NGC 6822, Online Material p 8
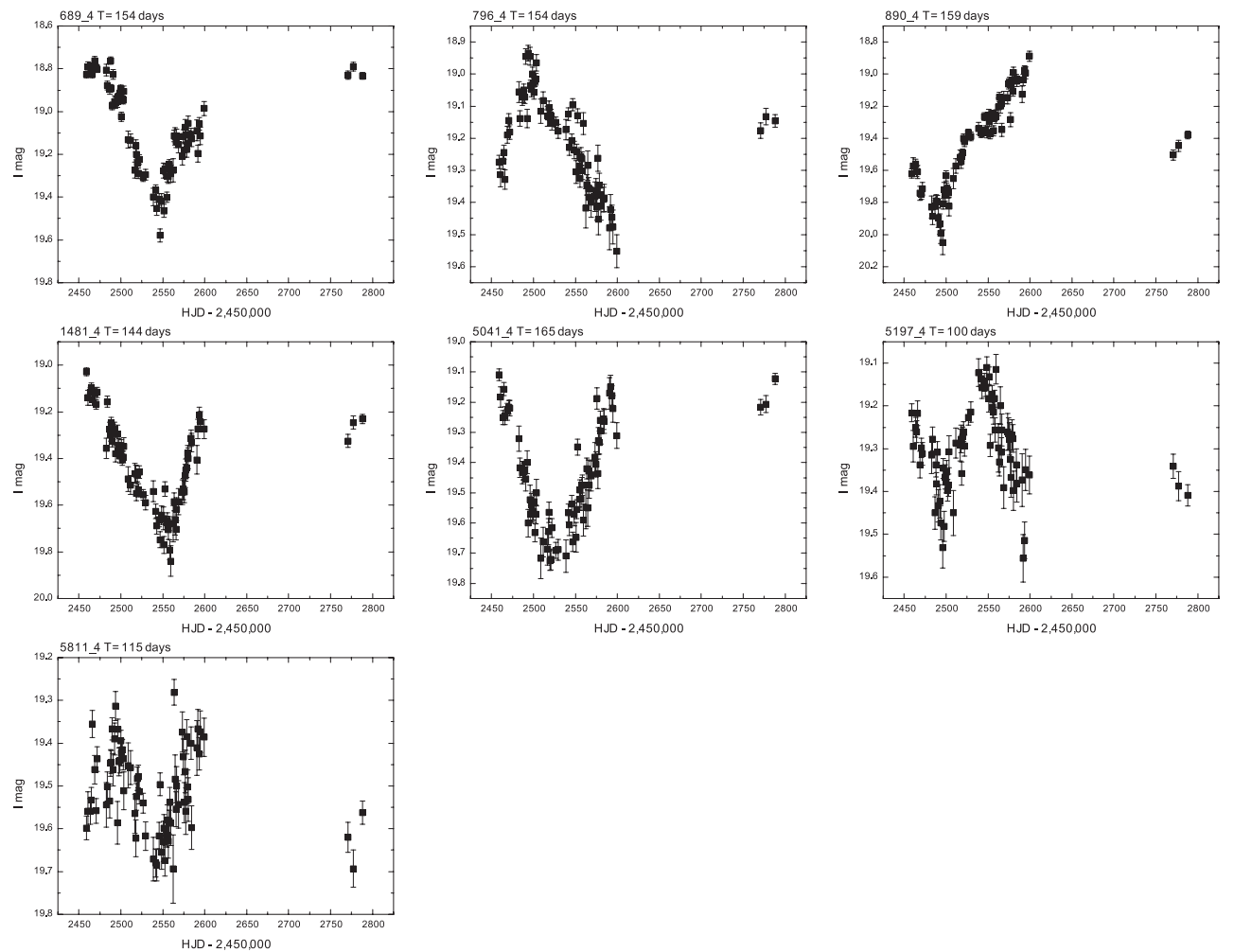

Fig. 2. continued.

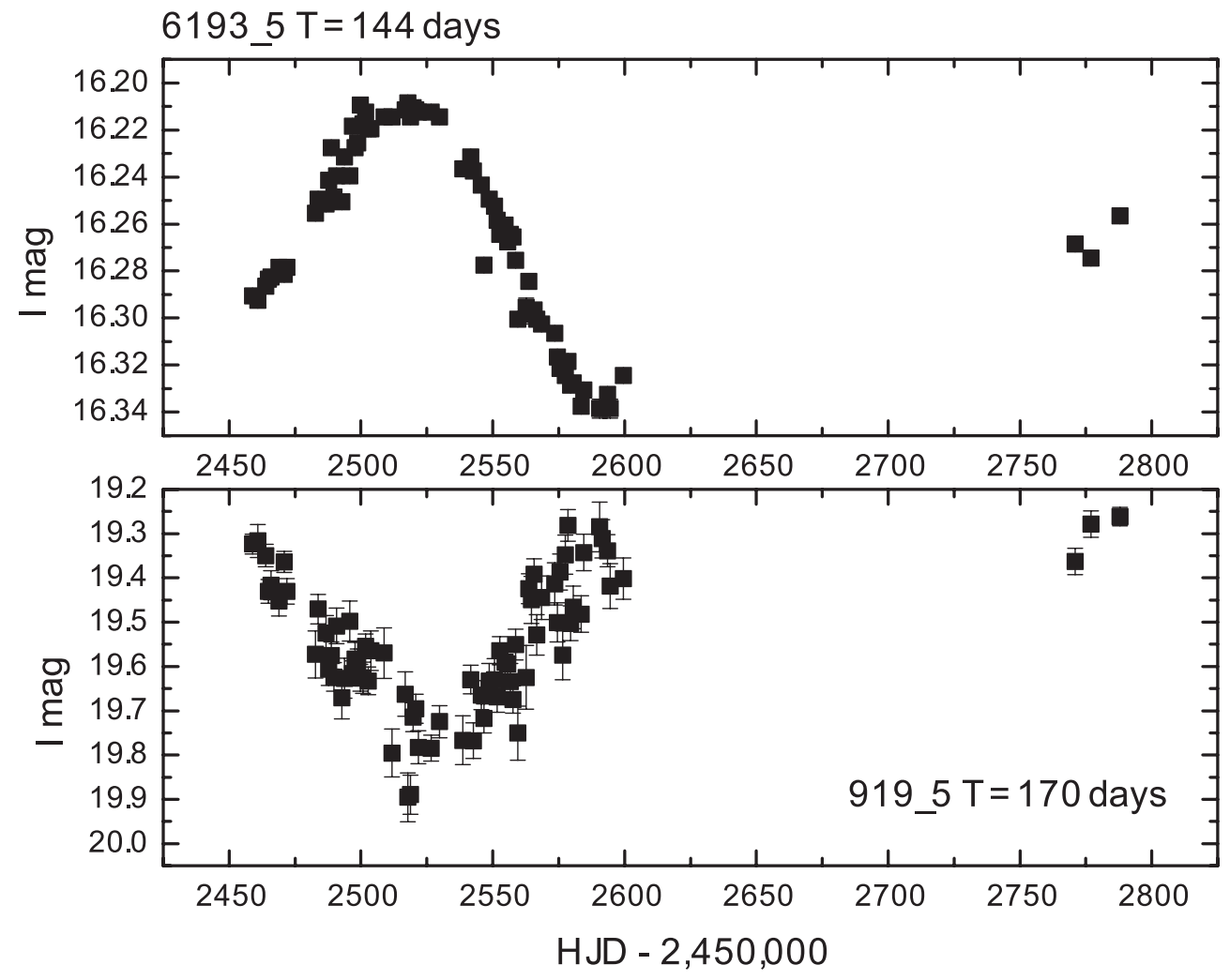

Fig. 2. continued. 
R. E. Mennickent et al.: Bright variable stars in NGC 6822, Online Material p 9
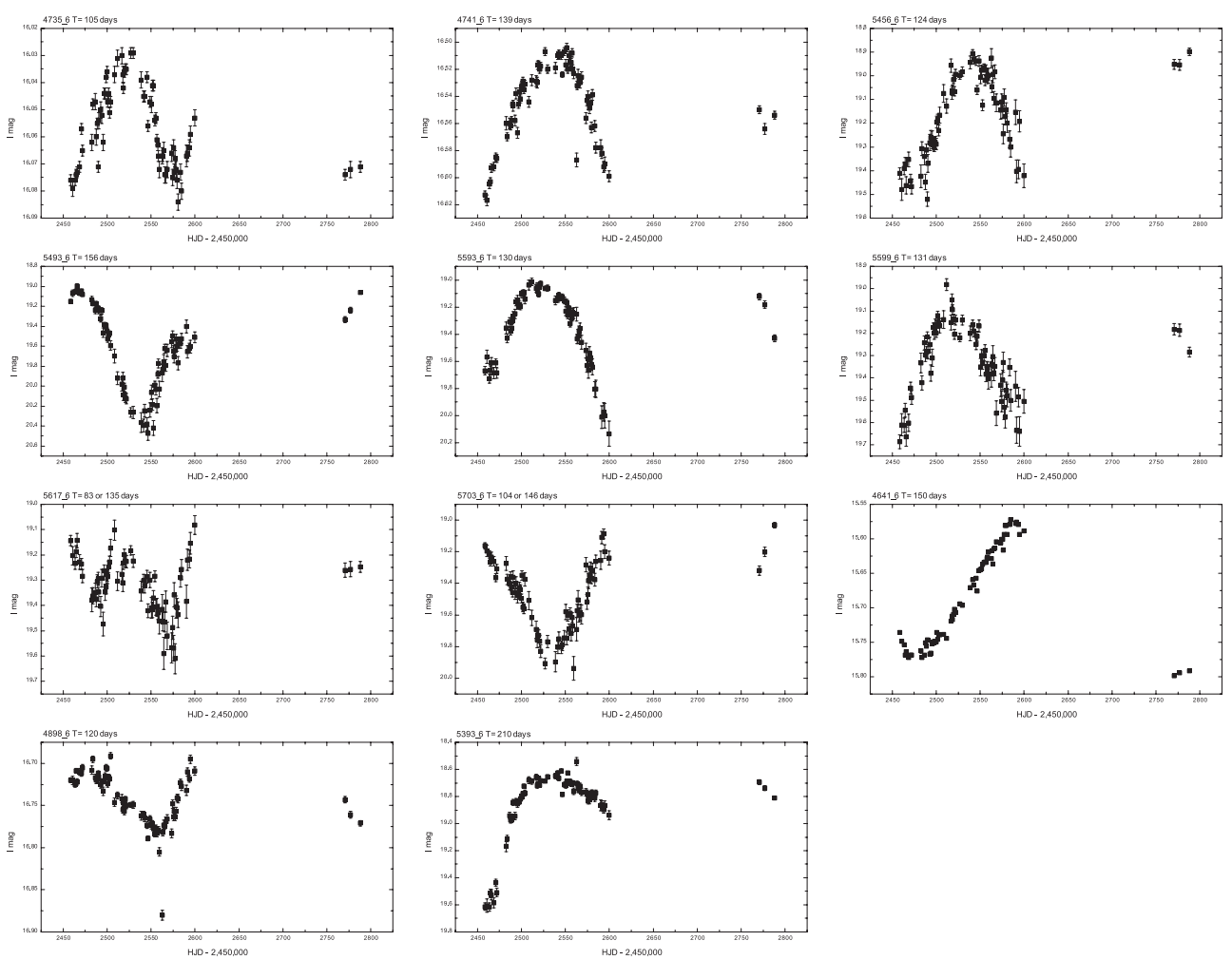

Fig. 2. continued.
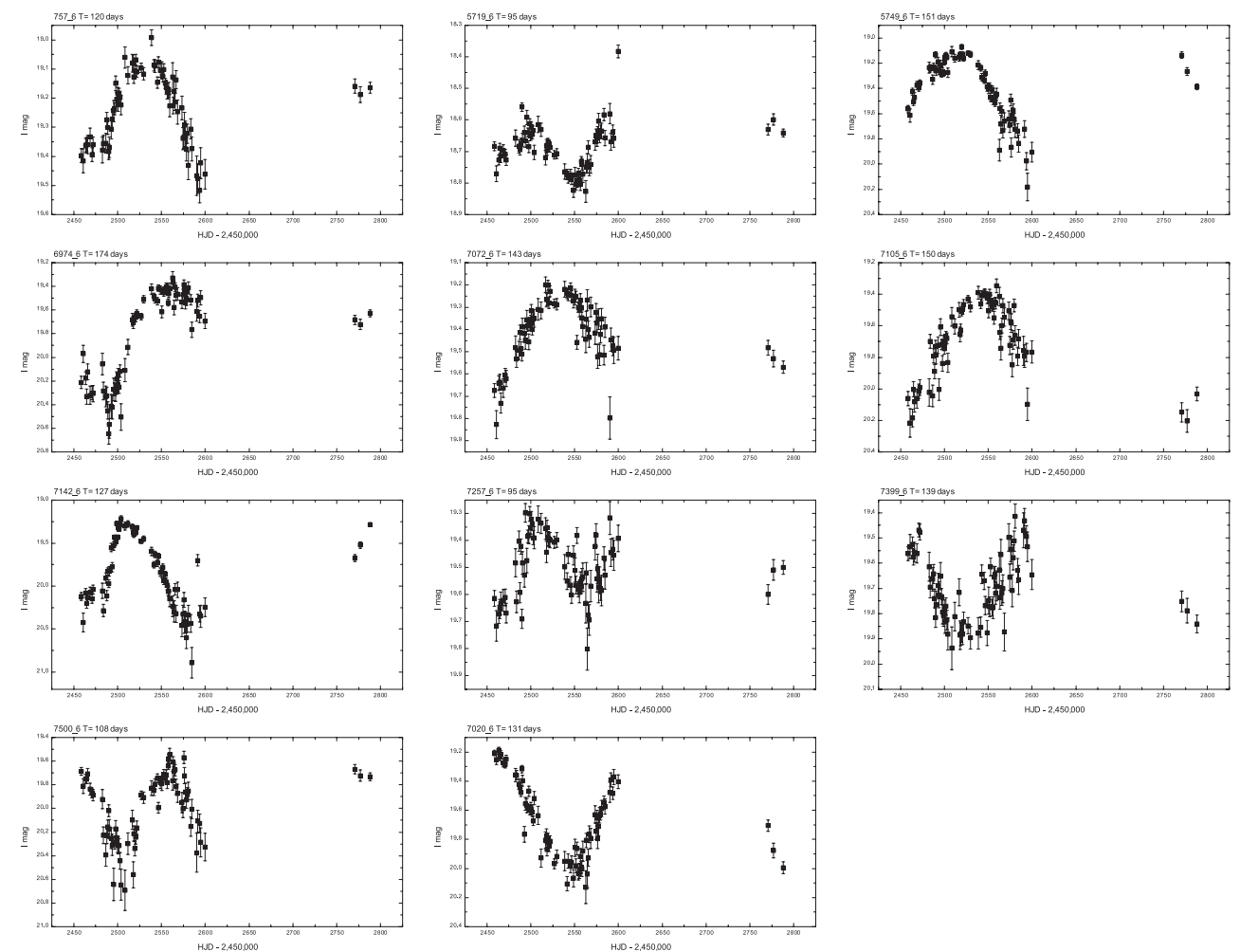

Fig. 2. continued. 
R. E. Mennickent et al.: Bright variable stars in NGC 6822, Online Material p 10

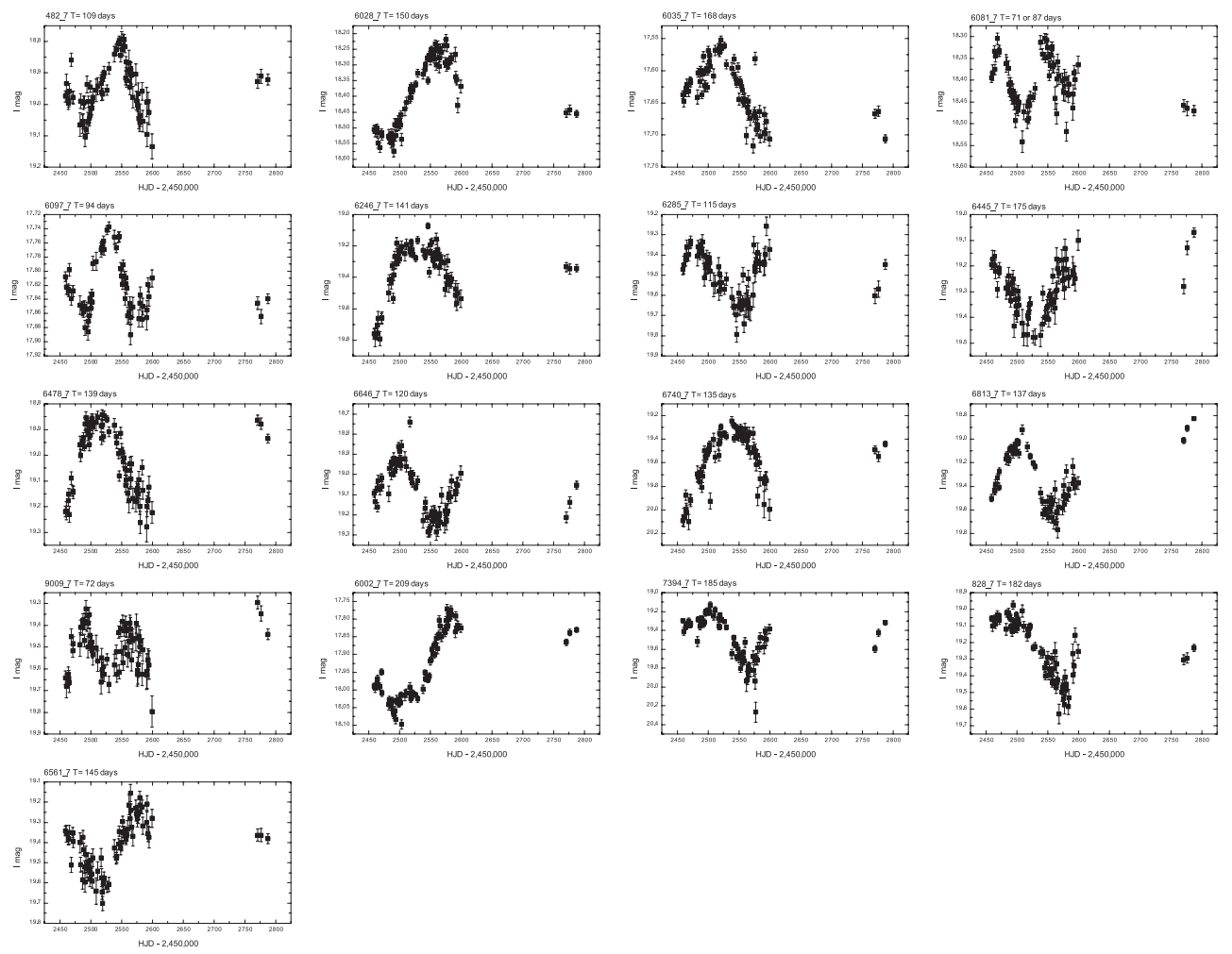

Fig. 2. continued.
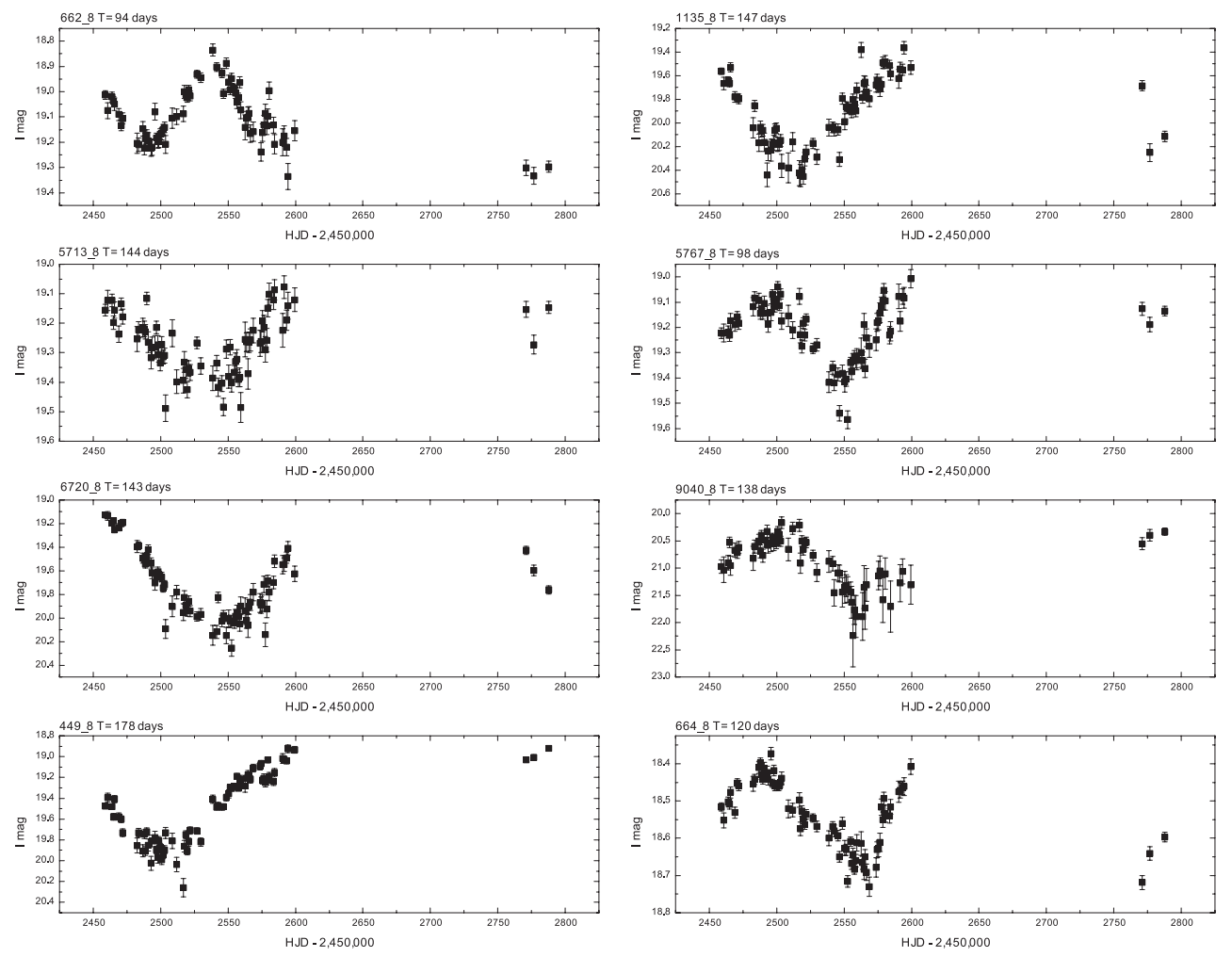

Fig. 2. continued. 